\title{
Dual Representation of the Cost of Designing a Portfolio Satisfying Multiple Risk Constraints. ${ }^{* \dagger}$
}

\author{
Géraldine Bouveret ${ }^{\ddagger}$ \\ University of Oxford \\ Smith School \& Wadham College \\ geraldine.bouveret@smithschool.ox.ac.uk
}

\begin{abstract}
We consider, within a Markovian complete financial market, the problem of finding the least expensive portfolio process meeting, at each payment date, three different types of risk criterion. Two of them encompass an expected utility-based measure and a quantile hedging constraint imposed at inception on all the future payment dates, while the other one is a quantile hedging constraint set at each payment date over the next one. The quantile risk measures are defined with respect to a stochastic benchmark and the expected utility-based constraint is applied to random payment dates. We explicit the Legendre-Fenchel transform of the pricing function. We also provide, for each quantile hedging problem, a backward dual algorithm allowing to compute their associated value function by backward recursion. The algorithms are illustrated with a numerical example.
\end{abstract}

Keywords: Stochastic Target Problems, Risk Constraints, Quantile Hedging, Expected Utility, Bermudan Options.

AMS 2010 Subject Classification Primary, 91G20, 91G60 ; Secondary, 60J60, 49L20.

\footnotetext{
*Part of this work has been written during the author's PhD at Imperial College London sponsored by the Natixis Foundation for Research and Innovation and during the author's visit to the Department of Economics of the University of Verona in February 2018 under the YITP Research Prize made available by ACRI (Association of Bank Foundations) within the Young Investigator Training Program (YITP).

${ }^{\dagger}$ The Version of Record of this manuscript has been published and is available in APPLIED MATHEMATICAL FINANCE 24/07/2019 http://www.tandfonline.com/10.1080/1350486X.2019.1638276.

${ }^{\ddagger}$ Current affiliation: Centre d’Economie de la Sorbonne, Université Paris 1 Panthéon Sorbonne, geraldine.bouveret@univ-paris1.fr

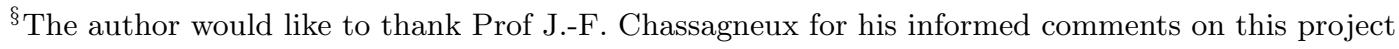
and Dr L. Moreau for his insight on Section 6 and especially on Theorem 6.1. The author is also grateful to the anonymous referees for their valuable remarks. All the remaining errors are the author's ones.
} 


\section{Introduction}

We face an economic context in which financial institutions are asked by regulators to secure enough capital to cover part of the risks they take. In particular, the involved authorities define a probabilistic risk criterion to measure the potential losses stemming from not being able to cover the future random liabilities. They determine accordingly the amount of assets to be secured by financial institutions to limit their debts (see e.g. the Basel II framework or the European Solvency II directive). The problem of asset-liability management under some risk targets has attracted a lot of interests (see e.g. Brandt and Van Binsbergen [8], Detemple and Rindisbacher [9] and Martellini and Milhau [18]). It is, in particular, relevant for pension funds coping with the longevity risk and the variable annuity-type feature embedded in their claims; and for fund managers dealing with stranded assets whose future value is uncertain.

Motivated by these issues, we first consider the problem of finding the cheapest portfolio process allowing to cover future random liabilities with a given degree of confidence. The involved risk criterion can be related to the probability of success of a devised hedge, laying the foundations of quantile hedging problems. More precisely, we want to compute the initial value of a portfolio process for which we can find a financial strategy such that it dominates a set of random liabilities with some probability. The computed initial value is therefore the cost of designing such portfolio and will be referred to as hedging price. Beyond the regulatory scope previously introduced, this question also arises when investors have a limited capital and want to design the most efficient strategies to profit from business opportunities while keeping the associated downside risks under control. Additionally and in the same spirit, this study is also interested in computing the minimal cost of designing a portfolio satisfying given levels of expected utility involving a deterministic benchmark above which the portfolio process must stay.

We therefore suggest three different formulations of the risk constraint: a Europeanstyle and next-period quantile hedging constraint and an American-style expected utility-based one (using the terminology of Jiao, Klopfenstein and Tankov [16]). In the first two cases, the constraint is imposed on the probability of successfully hedging random claims (liabilities) at each discrete and deterministic payment date. This probability is set at the initial date in the first case and at the previous payment date in the second one. In these cases, the constraints must be satisfied on a set of pre-specified discrete dates in order to comply with practical concerns. In the third case, a bound is imposed at inception on the level of expected utility achieved by the portfolio process at each random payment date. In particular, the domain of definition of the utility 
function integrates a constant benchmark above which the portfolio process must always stay, and the latter function satisfies some regularity assumptions. It is actually an obstacle and regular counterpart of the problem studied by Bouchard, Bouveret and Chassagneux in [2] (see [2, Proposition 2.1]) in the case of deterministic (constant) threshold.

This paper is organised as follows. In Section 2 we discuss the existing literature in the field as well as the novelty of the present paper. Then in Section 3 we detail the problem framework. Finally, in Section 4 and Section 5 we study the European-style and next-period quantile hedging constraints while in Section 6 we study the Americanstyle expected utility-based constraint.

Notations: Let $d$ be a positive integer. Any vector $x$ of $\mathbb{R}^{d}$ is viewed as a column vector, unless otherwise stated. We denote by $|x|$ the Euclidean norm of $x$, and by $x^{\top}$ its transpose. We set $\mathbb{M}^{d}:=\mathbb{R}^{d \times d}$ and denote by $M^{\top}$ the transpose of $M \in \mathbb{M}^{d}$. For a given set $S$ we also denote by $S^{c}$ its complementary set. We write $\mathcal{O}_{+}^{d}:=(0, \infty)^{d}$ to ease notations, and we fix a finite time horizon $T>0$. Let $\psi: p \in \mathbb{R} \mapsto \psi(p)$. The function $q \in \mathbb{R} \mapsto \psi^{\sharp}(q)$ is the Legendre-Fenchel transform (or the dual) of $p \mapsto \psi(p)$, if

$$
\psi^{\sharp}(q):=\sup _{p \in \mathbb{R}}(p q-\psi(p)),
$$

(if $\psi: p \in \mathbb{R}^{d} \mapsto \psi(p)$, we define similarly for $q \in \mathbb{R}^{d}, \psi^{\sharp}(q):=\sup _{p \in \mathbb{R}^{d}}\left(p^{\top} q-\psi(p)\right.$ ) unless otherwise stated). The variable $q$ is the dual of $p$. Moreover if $\psi$ is convex in its $p$ variable, we also denote by $\mathrm{D}_{p}^{+} \psi$ and $\mathrm{D}_{p}^{-} \psi$ its corresponding right- and leftderivatives. We refer to [20] for the various notions related to convex analysis. If $\psi$ is differentiable, we simply write $\mathrm{D}_{p} \psi$. Additionally, we introduce for a given function $\phi$ defined on $B \subseteq \mathbb{R}$ the set $\operatorname{Im}(\phi):=\{\phi(r), r \in B$ s.t. $\phi(r) \in \mathbb{R}\}$. We finally fix a complete probability space $(\Omega, \mathbb{F}, \mathbb{P})$ supporting a $d$-dimensional Brownian motion $W$. We denote by $\mathbb{F}=\left(\mathcal{F}_{t}\right)_{0 \leqslant t \leqslant T}$ the usual augmented Brownian filtration, and we introduce $\mathcal{T}_{[s, T]}$ the set of stopping times with values in $[s, T], 0 \leqslant s \leqslant T$. Similarly we define $\mathcal{T}_{[\tau, T]}$ with $\tau \in \mathcal{T}_{[s, T]}$, the set of stopping times $\theta$ such that for all $\omega \in \Omega, \tau(\omega) \leqslant \theta(\omega) \leqslant T$. Throughout the paper, inequalities between random variables have to be understood in the $\mathbb{P}$-a.s. sense. As usual, the abbreviation "s.t." stands for "such that". In this manuscript, the constant $C>0$ is a generic denomination of a constant factor unless otherwise stated. 


\section{Related literature \& novelty}

In the literature, the problem of hedging a European-type random liability under both probabilistic and expected shortfall constraints has been initiated by Föllmer and Leukert [14, 15]. They worked under a non-Markovian setting and within both complete and incomplete markets. They derived explicit solutions in a Black-Scholes setting. A similar question but in a Markovian setting arose with Soner and Touzi [21, 22] and Bouchard, Elie and Touzi [4]. Their arguments relied on stochastic control, viscosity solution and stochastic target theory. Bouchard, Elie and Réveillac [3], and later Dumitrescu [10] extended the previous results to the non-Markovian case. Moreover, some extensions to other classes of stochastic target problems have been performed (see e.g. Bouchard and $\mathrm{Vu}$ [6], Moreau [19] and Bouchard, Moreau and Nutz [5] for a Markovian setting, and [16] for a non-Markovian one). A related strain of the literature focuses on portfolio management under the constraint of outperforming a deterministic benchmark on a set of deterministic future dates (see e.g. El Karoui, Jeanblanc and Lacoste [13]) or a stochastic benchmark at a given date (see e.g. Boyle and Tian [7]). However, the problem of partially hedging multiple stochastic benchmarks or satisfying multiple and various risk criteria still lays challenges. A study of different shortfall constraints on a set of discrete and deterministic future dates has been pursued in [16]. Those constraints took the form of general lookback-style, next-period and European-style constraints in expectation set at the different exercise times. The authors worked with loss functions satisfying some regularity assumptions. However, the irregular loss function counterparts still raise issues. The probabilistic constraint involves an indicator function which is an archetype of an irregular loss function. Bouchard, Bouveret and Chassagneux thus extended in [2] the previous results on lookback-style constraints to the case where the constraints depend on the whole trajectory of the agent's portfolio and hold in probability. They proved that the latter problem reverted to a stochastic target problem of Bermudan type (see [2, Proposition 2.2]), which is a weak and discrete version of the problem studied in [6].

We suggest here to study the irregular loss-function counterpart of the Europeanstyle and next-period constraints considered in [16]. In addition, we consider the problem of computing the cost associated with the design of a portfolio always outperforming a constant benchmark and satisfying an American expected utility-based constraint defined from this benchmark. This problem has received little attention in the literature and our study extends the results in [13]. This paper therefore provides the dual representation of the initial price of those multi-objective risk-management problems. One of the key tools is to reduce, as in [4], the constraints defined in a weak (probabilistic) 
form to usual almost-sure ones. This is done at the price of enlarging the state space with (an) additional process(es) involving (an) unbounded control(s). In particular, in the case where the constraints hold in probability, a backward dual algorithm is derived to compute the value function using a backward recursion.

The analysis conducted in this manuscript is partially based on the results proved in [2] and the mathematical contribution of this paper is twofold: the proof of the dual algorithm as an upper bound to the hedging price under European-type quantile hedging constraints (see Theorem 4.1); and the handling of random times in the study of the cost of designing a portfolio satisfying an American expected utility-based constraint, impacting the conditions under which an explicit formulation can be found (see the problem statement in Section 6 and Theorem 6.1). In particular, the dual algorithm in Section 4 is more involved than the one studied in [2] as its structure must account for as many Legendre-Fenchel transforms as constraints holding from the payment date under interest until the terminal date. This raises difficulties in the representation and study of the properties (differentiability, limits, bounds, and continuity) of the dual algorithm. As a result, we only prove the algorithm on two successive dates to keep the computation tractable (see Proposition 4.3), and to allow for the derivation of a closed-form representation of the hedging price. We recall that, on the contrary, the dual algorithm derived in [2] only involves one Legendre-Fenchel transform at each payment date. We also underline that the derivation of the backward dual algorithm allowing to compute the hedging prices under next-period quantile hedging constraints (see Theorem 5.1) turns out to be the easiest problem to handle. This algorithm indeed involves only two successive dates and can be built from the one derived in the case of a single European constraint. This analysis therefore follows from Section 4. However, for the sake of completeness of the study of hedging prices under quantile hedging constraints, we find it important to add this result still missing in the literature.

The main results are derived under a Markovian and complete market framework, in the spirit of [2]. The market completeness assumption suggests that the randomness of the future liabilities, if any, is driven by "hedgeable" risk factors, such as the market risk. Finally, we argue by duality and thus only use probabilistic arguments opening the door to the study of more general non-Markovian settings. 


\section{Problems framework}

We consider the following market framework. The financial market is composed of $d$ risky assets $X=\left(X^{1}, \ldots, X^{d}\right)^{\top}$ with the following dynamics

$$
X_{s}^{t, x}=x+\int_{t}^{s} \mu\left(r, X_{r}^{t, x}\right) \mathrm{d} r+\int_{t}^{s} \sigma^{\top}\left(r, X_{r}^{t, x}\right) \mathrm{d} W_{r},
$$

given the initial data $(t, x) \in[0, T] \times \mathcal{O}_{+}^{d}$, and a non-risky asset, whose price process is normalized to unity. The coefficients of the diffusion are such that the process $X^{t, x}$ is the unique and strong $\mathcal{O}_{+}^{d}$-valued solution of (3.1) when the original data lies in $\mathcal{O}_{+}^{d}$. In particular

$$
\mu:[0, T] \times \mathcal{O}_{+}^{d} \rightarrow \mathbb{R}^{d} \text { and } \sigma:[0, T] \times \mathcal{O}_{+}^{d} \rightarrow \mathbb{M}^{d},
$$

are Lipschitz continuous in the space variable uniformly in the time variable.

To ensure the absence of arbitrage and the completeness of the financial market, we work under the following standing assumption.

Assumption 3.0.1.

$$
\sigma \text { is invertible and } \lambda:=\sigma^{-1} \mu \text { is bounded. }
$$

We therefore define the risk neutral measure ${ }^{1}$

$$
\mathbb{Q}_{t, x}:=\frac{1}{Q_{T}^{t, x, 1}} \cdot \mathbb{P} \text { with } \frac{1}{Q^{t, x, q}}:=\frac{1}{q} \mathcal{E}\left(-\int_{t} \lambda\left(s, X_{s}^{t, x}\right)^{\top} \mathrm{d} W_{s}\right), q>0 .
$$

Moreover we also consider that

$\lambda$ is Lipschitz continuous in space, uniformly in time,

which will be useful in some of our upcoming arguments.

We introduce two sets of admissible financial strategies

- $\mathcal{U}_{t, x, y}$ : the set of $d$-dimensional predictable processes $\nu$ valued in $\mathbb{R}^{d}$ such that for $(t, x, y) \in[0, T] \times \mathcal{O}_{+}^{d} \times \mathbb{R}^{+}$,

$$
\mathbb{E}^{\mathbb{Q}_{t, x}}\left[\int_{t}^{T}\left|\nu_{s}^{\top} \sigma\left(s, X_{s}^{t, x}\right)\right|^{2} d s\right]<\infty,
$$

and the corresponding wealth process remains non-negative

$$
Y^{t, x, y, \nu}:=y+\int_{t}^{\cdot} \nu_{r}^{\top} \mathrm{d} X_{r}^{t, x} \geqslant 0, \text { on }[t, T] ;
$$

\footnotetext{
${ }^{1} \mathcal{E}$ denotes here the Doléans-Dade exponential.
} 
- $\tilde{\mathcal{U}}_{t, x, y}^{K}$ : the set of $d$-dimensional predictable processes $\nu$ valued in $\mathbb{R}^{d}$ such that for $(t, x, y) \in[0, T] \times \mathcal{O}_{+}^{d} \times[K, \infty)$, for some $K \in \mathbb{R},(3.6)$ holds and the corresponding wealth process

$$
Y^{t, x, y, \nu}:=y+\int_{t} \nu_{r}^{\top} \mathrm{d} X_{r}^{t, x} \geqslant K, \text { on }[t, T]
$$

The set $\mathcal{U}_{t, x, y}$ will be used in both Section 4 and Section 5 while the set $\tilde{\mathcal{U}}_{t, x, y}^{K}$ will be used in Section 6. As usual, each $\nu_{t}^{i}$ should be interpreted as the number of units of asset $i$ in the portfolio at time $t$.

Moreover, we introduce $\mathcal{A}$, the set of square integrable predictable processes $\alpha$ valued in $\mathbb{R}^{d}$, and we define for $(t, u, p) \in[0, T] \times[t, T] \times[0,1]$ the subset

$$
\mathcal{A}_{t, p}^{u}:=\left\{\alpha \in \mathcal{A} \text { s.t. } P^{t, p, \alpha}:=p+\int_{t} \alpha_{v}^{\top} \mathrm{d} W_{v} \in[0,1] \text { on }[t, u]\right\} .
$$

This set will be used in both Section 4 and Section 5. We introduce a non-negative payoff function $g$ satisfying for $\left(t, x, x^{\prime}\right) \in[0, T] \times\left(\mathcal{O}_{+}^{d}\right)^{2}$,

$$
\begin{gathered}
|g(t, x)| \leqslant C(1+|x|), \text { for some } C>0, \\
\left|g(t, x)-g\left(t, x^{\prime}\right)\right| \leqslant C\left|x-x^{\prime}\right|, \text { for some } C>0 .
\end{gathered}
$$

Before ending this section we introduce two functions that will be of great interest in both Section 4 and Section 5. More precisely, we fix $n \in \mathbb{N}$ and consider the time grid $t_{0}=0 \leqslant \cdots \leqslant t_{i} \leqslant \cdots \leqslant t_{n}=T$ and introduce for $(x, p) \in \mathcal{O}_{+}^{d} \times \mathbb{R}$ the notations

$$
g\left(t_{i}, x, p\right):=g\left(t_{i}, x\right) \mathbf{1}_{\{0<p \leqslant 1\}}+\infty \mathbf{1}_{\{p>1\}} .
$$

We now introduce a function $f\left(t_{i}, \cdot\right): \mathcal{O}_{+}^{d} \times \mathbb{R}^{n-i} \rightarrow \mathbb{R}^{+}$being such that $f(T, \cdot)=0$. We are now able to define on $\mathcal{O}_{+}^{d} \times \mathbb{R}^{n-i+1}$,

$$
(f \vee g)\left(t_{i}, x, p_{i}, \ldots, p_{n}\right):=\left\{\begin{array}{l}
f\left(t_{i}, x, p_{i+1}, \ldots, p_{n}\right) \vee g\left(t_{i}, x, p_{i}\right), \text { if } i<n \\
g\left(t_{n}, x, p_{n}\right), \text { if } i=n
\end{array},\right.
$$

together with its closed convex envelope in the third variable,

$$
\begin{aligned}
& \overline{\operatorname{conv}}(f \vee g)\left(t_{i}, x, p_{i}, \ldots, p_{n}\right) \\
& :=\left\{\begin{array}{lc}
\left(\left[g\left(t_{i}, x\right)-f\left(t_{i}, x, p_{i+1}, \ldots, p_{n}\right)\right]^{+}\left[p_{i}\right]^{+}+f\left(t_{i}, x, p_{i+1}, \ldots, p_{n}\right)\right) \mathbf{1}_{\left\{p_{i} \leqslant 1\right\}} & +\infty \mathbf{1}_{\left\{p_{i}>1\right\}}, \\
p_{n} g\left(t_{n}, x\right) \mathbf{1}_{\left\{0 \leqslant p_{n} \leqslant 1\right\}}+\infty \mathbf{1}_{\left\{p_{n}>1\right\}}, \text { if } i=n & \text { if } i<n
\end{array} .\right.
\end{aligned}
$$


Finally we denote by $v_{\mathrm{BM}}$ the problem studied in [2], i.e. for $(t, x, p) \in[0, T] \times \mathcal{O}_{+}^{d} \times$ $[0,1]$,

$$
v_{\mathrm{BM}}(t, x, p):=\inf \Gamma_{\mathrm{BM}}(t, x, p),
$$

where

$$
\Gamma_{\mathrm{BM}}(t, x, p):=\left\{y \geqslant 0: \exists \nu \in \mathcal{U}_{t, x, y} \text { s.t. } \mathbb{P}\left[\bigcap_{s \in \mathbb{T}_{t}} \mathcal{S}_{s}^{t, x, y, \nu}\right] \geqslant p\right\}
$$

with

$$
\mathcal{S}_{s}^{t, x, y, \nu}:=\left\{\begin{array}{lll}
\Omega & \text { if } & s \leqslant t \\
\left\{Y_{s}^{t, x, y, \nu} \geqslant g\left(s, X_{s}^{t, x}\right)\right\} & \text { if } & s>t
\end{array},\right.
$$

and

$$
\mathbb{T}_{t}:=\left\{t_{0}=0 \leqslant \cdots \leqslant t_{i} \leqslant \cdots \leqslant t_{n}=T\right\} \cap(t, T] .
$$

We finally recall [2, eq. (2.8)],

$$
v_{\mathrm{BM}}(t, x, 1)=\mathbb{E}^{\mathbb{Q} t, x}\left[\left(v_{\mathrm{BM}} \vee g\right)\left(t_{i+1}, X_{t_{i+1}}^{t, x}, 1\right)\right] \text {, for } t \in\left[t_{i}, t_{i+1}\right), i<n .
$$

Remark 3.1. Assume that the constraint stated in (3.13) holds at the terminal date only. Interestingly enough, we can easily link the VaR constraint to the quantile hedging one. Indeed assume that $L$ is the VaR limit for the time horizon T. The usual VaR constraint on a portfolio is $\mathbb{P}[$ Loss $\geqslant L] \leqslant \beta$ where Loss $:=y-Y_{T}^{t, x, y, \nu}$ and $\beta$ is the confidence level. This VaR constraint is equivalent to $\mathbb{P}\left[Y_{T}^{t, x, y, \nu} \geqslant y-L\right] \geqslant 1-\beta$. Taking $L=y-g\left(T, X_{T}^{t, x}\right)$ we recover our quantile hedging constraint. Moreover, as noted in [14], the amount of capital required to reach the target will be less than in the static VaR approach as we allow for dynamic strategies. This remark obviously holds for (4.1) and (5.1) studied below.

\section{Problem 1: Hedging price under European quantile hedging constraints}

We consider the hedging problem under a family of European quantile hedging constraints. This problem is more involved than the one studied in [2] as each constraint holds from inception until the corresponding payment date (see Proposition 4.2). The structure of the backward dual algorithm is thus more complex. Indeed, at each payment date, it will account for as many Legendre-Fenchel transforms as constraints holding from the latter payment date until the terminal date (see Theorem 4.1). We will 
therefore only prove the algorithm on two successive dates in order to keep the computation tractable (see Proposition 4.3), and to be able to derive closed-form representation of the hedging price.

Let us define the problem we intend to solve.

On $\left[t_{i}, t_{i+1}\right) \times \mathcal{O}_{+}^{d} \times[0,1]^{n-i}, 0 \leqslant i \leqslant n-1$ we define

$$
v_{\mathrm{EU}}\left(t, x, p_{i+1}, \ldots, p_{n}\right):=\inf \Gamma_{\mathrm{EU}}\left(t, x, p_{i+1}, \ldots, p_{n}\right),
$$

with

$$
\Gamma_{\mathrm{EU}}\left(t, x, p_{i+1}, \ldots, p_{n}\right):=\left\{y \geqslant 0: \exists \nu \in \mathcal{U}_{t, x, y} \text { s.t. } \mathbb{P}\left[\mathcal{S}_{t_{k}}^{t, x, y, \nu}\right] \geqslant p_{k}, i+1 \leqslant k \leqslant n\right\},
$$

and where we use the same notation as in (3.14), i.e. $\mathcal{S}_{t_{k}}^{t, x, y, \nu}:=\left\{Y_{t_{k}}^{t, x, y, \nu} \geqslant g\left(t_{k}, X_{t_{k}}^{t, x}\right)\right\}, k \geqslant$ $i+1$. On $\mathcal{O}_{+}^{d} \times[0,1]$, we set $v_{\mathrm{EU}}(T, \cdot)=0$.

Remark 4.1. On $\left[t_{i}, t_{i+1}\right) \times \mathcal{O}_{+}^{d}, 0 \leqslant i \leqslant n-1, v_{\mathrm{EU}}\left(\cdot, p_{i+1}, \ldots, p_{n}\right)$ is equal to 0 if $p_{k} \leqslant p_{\min }^{k}(t, x), i+1 \leqslant k \leqslant n$, where

$$
p_{\min }^{k}(t, x):=\mathbb{P}\left[g\left(t_{k}, X_{t_{k}}^{t, x}\right)=0\right]<1,
$$

by assumption. We use the convention $p_{\min }^{n}(T, \cdot)=1$ on $\mathcal{O}_{+}^{d}$. In particular, on $\mathcal{O}_{+}^{d}$,

$$
v_{\mathrm{EU}}(t, \cdot, 1, \ldots, 1)>0, \text { for } t<T \text {. }
$$

As in [2] we can restrict to strategies such that $v_{\mathrm{EU}}$ satisfies a linear growth. This is made clear in the subsequent remark.

Remark 4.2. It follows from (3.8) that we can restrict the admissible strategies $\nu$ to those satisfying for $(t, x, y) \in[0, T] \times \mathcal{O}_{+}^{d} \times \mathbb{R}^{+}$,

$$
0 \leqslant Y^{t, x, y, \nu} \leqslant C\left(1+\left|X^{t, x}\right|\right), C>0,
$$

by appealing to a buy-and-hold strategy after the first time at which the wealth process hits the term in the right-hand side of the previous inequality (recall that $X^{t, x}$ has components valued in $(0, \infty))$. Therefore on $\left[t_{i}, t_{i+1}\right) \times \mathcal{O}_{+}^{d} \times[0,1]^{n-i}, 0 \leqslant i \leqslant n-1$,

$$
0 \leqslant v_{\mathrm{EU}}\left(t, x, p_{i+1}, \ldots, p_{n}\right) \leqslant C(1+|x|) .
$$

Similarly to [2], we intend to build a backward dual representation of $v_{\mathrm{EU}}$.

We can already make the following remark on the relationship between the value function $v_{\mathrm{BM}}$ defined in (3.13) and $v_{\mathrm{EU}}$. 


\section{Remark 4.3.}

(a) On $\left[t_{n-1}, t_{n}\right) \times \mathcal{O}_{+}^{d}, v_{\mathrm{BM}}\left(t, x, p^{\prime}\right)=v_{\mathrm{EU}}\left(t, x, p^{\prime}\right), p^{\prime} \in(-\infty, 1]$. Moreover on $\mathcal{O}_{+}^{d} \times$ $[0,1], v_{\mathrm{BM}}(T, \cdot)=v_{\mathrm{EU}}(T, \cdot)=0$.

(b) On $\left[t_{i}, t_{i+1}\right) \times \mathcal{O}_{+}^{d}, 0 \leqslant i \leqslant n-1, v_{\mathrm{BM}}(t, x, 1)=v_{\mathrm{EU}}(t, x, 1, \ldots, 1), v_{\mathrm{BM}}(t, x, p)=$ $v_{\mathrm{EU}}\left(t, x, p_{i+1}, \ldots, p_{n}\right)$ whenever $\left(p, p_{i+1}, \ldots, p_{n}\right) \in(-\infty, 0]^{n-i+1}$ and if there exists a $i+1 \leqslant$ $k \leqslant n$ for which $p_{k}>1, v_{\mathrm{BM}}(t, x, p)=v_{\mathrm{EU}}\left(t, x, p_{i+1}, \ldots, p_{n}\right)=\infty$ whenever $p>1$.

We now proceed as in [2] to represent the value function $v_{\mathrm{EU}}$. In Proposition 4.1, we convert the initial problem into a stochastic target one of an American type (see [6]). This result is fundamental to derive a preliminary probabilistic representation of $v_{\mathrm{EU}}$ in Proposition 4.2. We will then dedicate the rest of this section to the construction of a backward dual algorithm to compute $v_{\mathrm{EU}}$ (see Theorem 4.1). The latter will be built on two successive dates only as at each payment date it involves as many Legendre-Fenchel transformations as constraints and thus becomes hardly tractable for more than two constraints.

\subsection{Equivalent formulation as a stochastic target problem and dy- namic programming}

We omit the proof of the subsequent problem reduction since it follows the same lines as those in [2, proof of Proposition 2.2].

Proposition 4.1. Fix $\left(t, x, p_{i+1}, \ldots, p_{n}\right) \in\left[t_{i}, t_{i+1}\right) \times \mathcal{O}_{+}^{d} \times[0,1]^{n-i}, 0 \leqslant i \leqslant n-1$, then $\Gamma_{\mathrm{EU}}\left(t, x, p_{i+1}, \ldots, p_{n}\right)=\left\{\begin{array}{c}y \geqslant 0: \exists\left(\nu, \alpha_{i+1}, \ldots, \alpha_{n}\right) \in \mathcal{U}_{t, x, y} \times \mathcal{A}_{t, p_{i+1}}^{t_{i+1}} \times \ldots \times \mathcal{A}_{t, p_{n}}^{t_{n}} \text { s.t. } \\ Y_{t_{k}}^{t, x, y, \nu} \geqslant g\left(t_{k}, X_{t_{k}}^{t, x}, P_{t_{k}}^{t, p_{k}, \alpha_{k}}\right), i+1 \leqslant k \leqslant n\end{array}\right\}$, with the notation of (3.10) and (3.7).

Again the formulation obtained in Proposition 4.1 is key to derive a first dynamic programming algorithm whose proof is omitted as it is standard (see [2, proof of Proposition 2.3]). It appeals in particular to (3.8)-(3.9) and (4.4).

Proposition 4.2. Fix $\left(t, x, p_{i+1}, \ldots, p_{n}\right) \in\left[t_{i}, t_{i+1}\right) \times \mathcal{O}_{+}^{d} \times[0,1]^{n-i}, 0 \leqslant i \leqslant n-1$,

$$
\begin{aligned}
& v_{\mathrm{EU}}\left(t, x, p_{i+1}, \ldots, p_{n}\right) \\
& =\left\{\begin{array}{ll}
\inf _{\left(\alpha_{i+1}, \ldots, \alpha_{n}\right) \in \mathcal{A}_{t, p_{i+1}}^{t_{i+1}} \times \ldots \times \mathcal{A}_{t, p_{n}}^{t_{n}}} \mathbb{E}^{\mathbb{Q}_{t, x}}\left[\left(v_{\mathrm{EU}} \vee g\right)\left(t_{i+1}, X_{t_{i+1}}^{t, x}, P_{t_{i+1}}^{t, p_{i+1}, \alpha_{i+1}}, \ldots, P_{t_{i+1}}^{t, p_{n}, \alpha_{n}}\right)\right], \\
\inf _{\alpha \in \mathcal{A}_{t, p_{n}}^{t_{n}}} \mathbb{E}^{\mathbb{Q}_{t, x}\left[g\left(t_{n}, X_{t_{n}}^{t, x}, P_{t_{n}}^{t, p_{n}, \alpha}\right)\right],}, \text { if } i<n-1
\end{array},\right.
\end{aligned}
$$


where we used the notations given by (3.11) with $f \equiv v_{\mathrm{EU}}$ and (3.7).

As a consequence, there exists a constant $C>0$ such that for all $\left(t, x, x^{\prime}\right) \in\left[t_{i}, t_{i+1}\right) \times$ $\left(\mathcal{O}_{+}^{d}\right)^{2}, 0 \leqslant i \leqslant n-1$ and $\left(p_{i+1}, \ldots, p_{n}\right) \in[0,1]^{n-i}$,

$$
\left|v_{\mathrm{EU}}\left(t, x, p_{i+1}, \ldots, p_{n}\right)-v_{\mathrm{EU}}\left(t, x^{\prime}, p_{i+1}, \ldots, p_{n}\right)\right| \leqslant C\left(1+|x|+\left|x^{\prime}\right|\right)\left|x-x^{\prime}\right| .
$$

Remark 4.4. We will prove in the following section that $\left(v_{\mathrm{EU}} \vee g\right)$ can be replaced by its convex envelope with respect to $\left(p_{1}, p_{2}\right)$ in (4.5) (see Proposition 4.4). This phenomenon has already been highlighted in [2] and [4, 3].

\subsection{Backward dual algorithm}

We can now state the main theorem of this part which allows to compute $v_{\mathrm{EU}}$ defined in Proposition 4.2. More precisely it will lead to a characterization of the optimum reached in (4.5). As mentioned at the beginning of the section we will focus on the case where $n=2$, i.e. $T=t_{2}$ as, in this case, the algorithm involves on $\left[t_{0}, t_{1}\right)$ two Legendre-Fenchel transformations which are still tractable (see Proposition 4.3).

We introduce on $\mathcal{O}_{+}^{d} \times \mathbb{R}$ and $\mathcal{O}_{+}^{d} \times \mathbb{R}^{2}$ the following algorithm

$$
\left\{\begin{array}{rll}
w_{\mathrm{EU}}(T, x, q) & := & q+\infty \mathbf{1}_{\{q<0\}} \\
w_{\mathrm{EU}}(t, x, q) & := & \mathbb{E}^{\mathbb{Q} t, x}\left[\left(w_{\mathrm{EU}}^{\sharp} \vee g\right)^{\sharp}\left(t_{2}, X_{t_{2}}^{t, x}, q Q_{t_{2}}^{t, x, 1}\right)\right]+\infty \mathbf{1}_{\{q<0\}}, \quad t \in\left[t_{1}, t_{2}\right), \\
w_{\mathrm{EU}}\left(t, x, q_{1}, q_{2}\right) & := & \mathbb{E}^{\mathbb{Q} t, x}\left[\left(w_{\mathrm{EU}}^{\sharp} \vee g\right)^{\sharp}\left(t_{1}, X_{t_{1}}^{t, x}, q_{1} Q_{t_{1}}^{t, x, 1}, q_{2} Q_{t_{1}}^{t, x, 1}\right)\right]+\infty \mathbf{1}_{\left\{q_{2}<0\right\} \cup\left\{q_{1}<0\right\},}, \\
& & t \in\left[t_{0}, t_{1}\right)
\end{array},\right.
$$

with $Q^{t, x, 1}$ defined in (3.4) and where for all $\left(x, q_{1}, q_{2}\right) \in \mathcal{O}_{+}^{d} \times\left(\mathbb{R}^{+}\right)^{2}$ the function

$$
\left(w_{\mathrm{EU}}^{\sharp} \vee g\right)^{\sharp}\left(t_{1}, x, q_{1}, q_{2}\right):=\sup _{\left(p_{1}, p_{2}\right) \in \mathbb{R}^{2}}\left\{p_{1} q_{1}+p_{2} q_{2}-\left(w_{\mathrm{EU}}^{\sharp} \vee g\right)\left(t_{1}, x, p_{1}, p_{2}\right)\right\},
$$

and for all $q \in \mathbb{R}^{+}$,

$$
\left(w_{\mathrm{EU}}^{\sharp} \vee g\right)^{\sharp}\left(t_{2}, x, q\right):=\sup _{p \in \mathbb{R}}\left\{p q-\left(w_{\mathrm{EU}}^{\sharp} \vee g\right)\left(t_{2}, x, p\right)\right\} .
$$

In particular, $w_{\mathrm{EU}}(t, x, q)=\mathbb{E}^{\mathbb{Q}_{t, x}}\left[\left[q Q_{T}^{t, x, 1}-g\left(T, X_{T}^{t, x}\right)\right]^{+}\right]+\infty \mathbf{1}_{\{q<0\}}$.

The aim of this section is to prove that, on $\mathcal{O}_{+}^{d} \times \mathbb{R}^{2},(4.7)$ is the proper algorithm to compute the value function $v_{\mathrm{EU}}^{\sharp}$ and thus $v_{\mathrm{EU}}$ on $\left[t_{0}, T\right]$. Our main result is therefore given by the subsequent theorem.

Theorem 4.1. On $\left[t_{0}, T\right] \times \mathcal{O}_{+}^{d} \times[0,1]^{2}, v_{\mathrm{EU}}=w_{\mathrm{EU}}^{\sharp}$. 
Remark 4.5. We know from Remark 4.3 (a) that $v_{\mathrm{EU}}=v_{\mathrm{BM}}$ on $\left[t_{1}, T\right] \times \mathcal{O}_{+}^{d} \times[0,1]$. Therefore it follows from [2] that the theorem already holds on $\left[t_{1}, T\right] \times \mathcal{O}_{+}^{d} \times[0,1]$ as $v_{\mathrm{BM}}=w_{\mathrm{EU}}^{\sharp}$ on that domain. In particular $p \mapsto v_{\mathrm{EU}}(t, x, p)$, is continuous for all $(t, x) \in\left[t_{1}, T\right] \times \mathcal{O}_{+}^{d}$ (see [2, Proposition 3.2]). Therefore, the main result of this study relies on proving that the dual representation actually holds on $\left[t_{0}, t_{1}\right) \times \mathcal{O}_{+}^{d} \times[0,1]^{2}$.

We must now verify that $\left(w_{\mathrm{EU}}^{\sharp} \vee g\right)$ behaves in a sufficiently nice way backward in time.

\subsection{Proof of the backward dual representation}

Using Remark 4.5 and the Legendre-Fenchel transform definition, we easily obtain $v_{\mathrm{EU}} \geqslant w_{\mathrm{EU}}^{\sharp}$ on $\left[t_{0}, T\right] \times \mathcal{O}_{+}^{d} \times[0,1]^{2}$ (see e.g. [2, proof of Proposition 3.1]). We will then only focus on the proof of the backward algorithm as an upper bound to $v_{\mathrm{EU}}$ on $\left[t_{0}, t_{1}\right) \times \mathcal{O}_{+}^{d} \times[0,1]^{2}$, by Remark 4.5. To this aim we have to study beforehand the representation and differentiability of the dual algorithm. This is the purpose of the next section.

\subsubsection{Representation and differentiability of the backward dual algorithm}

We now examine the function $\left(w_{\mathrm{EU}}^{\sharp} \vee g\right)^{\sharp}$, displayed in the dual algorithm (4.7), and its Legendre-Fenchel transform $\left(w_{\mathrm{EU}}^{\sharp} \vee g\right)^{\sharp \sharp}$. We therefore provide a decomposition in Proposition 4.3 and analyze, in Lemma 4.2 , the sub-differential of $w_{\mathrm{EU}}\left(t_{0}, \cdot\right)$ in terms of the sub-differential of $w_{\mathrm{EU}}\left(t_{1}, \cdot\right)$. These results will be involved in the final proof of Theorem 4.1. Indeed a particular value $\left(p_{1}, p_{2}\right)$ in the sub-differential of $w_{\mathrm{EU}}\left(t_{0}, \cdot\right)$ will have to be found and a martingale representation argument will be required to link the elements of the sub-differential of $\left(w_{\mathrm{EU}}^{\sharp} \vee g\right)^{\sharp}$ at $t_{1}$ to $\left(p_{1}, p_{2}\right)$ at $t_{0}$ (see the proof of Theorem 4.2 below).

The following remark is key to obtain the representation of $\left(w_{\mathrm{EU}}^{\sharp} \vee g\right)^{\sharp}$ and thus to prove Proposition 4.3.

Remark 4.6. We observe that for $\left(x, p_{2}\right) \in \mathcal{O}_{+}^{d} \times(-\infty, 1]$,

$$
F_{t_{1}, x, p_{2}}: q_{1} \in \mathbb{R}^{+} \mapsto-w_{\mathrm{EU}}^{\sharp}\left(t_{1}, x, p_{2}\right)+\left[q_{1}-\left[g\left(t_{1}, x\right)-w_{\mathrm{EU}}^{\sharp}\left(t_{1}, x, p_{2}\right)\right]^{+}\right]^{+},
$$

is actually equal to the Legendre-Fenchel transform of $\left(w_{\mathrm{EU}}^{\sharp} \vee g\right)\left(t_{1}, x, p_{1}, p_{2}\right)$ with respect to $p_{1}$. This is the function that we recover in the proof of Theorem 7.1 in the appendix (see (7.3)). 
With the previous Remark at hand we already understand that the second LegendreFenchel transform, i.e. the one with respect to $p_{2}$, will involve some computations as several cases will have to be considered depending on the values taken by $\left(x, q_{1}\right)$. We thus start with a result (see Lemma 4.1 below) that will be of important use in the proof of Proposition 4.3.

We first define for $(x, \ell) \in \mathcal{O}_{+}^{d} \times \mathbb{R}^{+}$,

$$
\tilde{g}\left(t_{1}, x, \ell\right):=g\left(t_{1}, x\right)-\ell .
$$

Then using the non-decreasing property of $p \mapsto w_{\mathrm{EU}}^{\sharp}\left(t_{1}, x, p\right), x \in \mathcal{O}_{+}^{d}$, on its domain $(-\infty, 1]$ (see Remark 4.5), we introduce

$$
\left\{\begin{array}{ll}
\tilde{p}\left(t_{1}, x, \ell\right):=\inf \left\{p \in \mathbb{R} \text { s.t. }: w_{\mathrm{EU}}^{\sharp}\left(t_{1}, x, p\right) \geqslant \tilde{g}\left(t_{1}, x, \ell\right)\right\} \wedge 1, & (x, \ell) \in \mathcal{O}_{+}^{d} \times \mathbb{R}^{+} \\
\hat{p}\left(t_{1}, x\right):=\inf \left\{p \in \mathbb{R} \text { s.t. }: w_{\mathrm{EU}}^{\sharp}\left(t_{1}, x, p\right) \geqslant g\left(t_{1}, x\right)\right\} \wedge 1, & x \in \mathcal{O}_{+}^{d}
\end{array} .\right.
$$

For the reader's convenience we will omit, in the following, the dependance of $\tilde{p}$ in $\left(t_{1}, x, \ell\right)$ and the dependence of $p_{\min }^{2}$ (recall the definition in (4.2)) and $\hat{p}$ in $\left(t_{1}, x\right)$.

Lemma 4.1. Fix $(x, \ell) \in \mathcal{O}_{+}^{d} \times(0, \infty)$. We assume $g\left(t_{1}, x\right)>0$. Moreover we introduce the function

$$
f:=f_{t_{1}, x, \ell}: p \mapsto w_{\mathrm{EU}}^{\sharp}\left(t_{1}, x, p\right)-\left[\ell-\left[g\left(t_{1}, x\right)-w_{\mathrm{EU}}^{\sharp}\left(t_{1}, x, p\right)\right]^{+}\right]^{+} \mathbf{1}_{\{p \leqslant 1\}}+\infty \mathbf{1}_{\{p>1\}} .
$$

(1) Assume that $p_{\min }^{2}<\tilde{p}<1$ and $\hat{p} \geqslant 1$.

(a) The closed convex envelope of $f$ is given by

$$
f^{\sharp \sharp}(p)=\overline{\operatorname{conv}}(f)(p)=a\left[p-p_{\min }^{2}\right]^{+} \mathbf{1}_{\{p \leqslant 1\}}+\infty \mathbf{1}_{\{p>1\}},
$$

with $a:=a_{t_{1}, x, \ell}:=\frac{\tilde{g}\left(t_{1}, x, \ell\right)}{1-p_{\min }^{2}}$.

(b) Moreover we have for $q \geqslant 0$,

$$
f^{\sharp}(q)=p_{\min }^{2} q \vee\left(q-\tilde{g}\left(t_{1}, x, \ell\right)\right),
$$

which is a closed proper convex function.

(2) Assume that $p_{\min }^{2}<\tilde{p}<1$ and $\tilde{p}<\hat{p}<1$.

(a) The closed convex envelope of $f$ is given by

$$
f^{\sharp \sharp}(p)=\overline{\operatorname{conv}}(f)(p)=\hat{a}\left[p-p_{\min }^{2}\right]^{+} \mathbf{1}_{\{p \leqslant \hat{p}\}}+\left(w_{\mathrm{EU}}^{\sharp}\left(t_{1}, x, p\right)-\ell\right) \mathbf{1}_{\{\hat{p}<p \leqslant 1\}}+\infty \mathbf{1}_{\{p>1\}},
$$


with $\hat{a}:=\hat{a}_{t_{1}, x, \ell}:=\frac{\tilde{g}\left(t_{1}, x, \ell\right)}{\hat{p}-p_{\min }^{2}}$.

(b) Moreover we have for $q \geqslant 0$,

$$
f^{\sharp}(q)=\left[p_{\min }^{2} q \vee\left(\hat{p} q-\tilde{g}\left(t_{1}, x, \ell\right)\right)\right] \mathbf{1}_{\left\{q \leqslant \mathrm{D}_{p}^{+} f(\hat{p})\right\}}+\left(w_{\mathrm{EU}}\left(t_{1}, x, q\right)+\ell\right) \mathbf{1}_{\left\{q>\mathrm{D}_{p}^{+} f(\hat{p})\right\}},
$$

which is a closed proper convex function. In particular, it is continuous at $\mathrm{D}_{p}^{+} f(\hat{p})$ when $0<\mathrm{D}_{p}^{+} f(\hat{p})<+\infty$.

Proof. We argue for all $(x, \ell) \in \mathcal{O}_{+}^{d} \times(0, \infty)$. First of all we notice that

$$
f(p)=f_{1}(p) \mathbf{1}_{\{p \leqslant 1\}}+\infty \mathbf{1}_{\{p>1\}},
$$

with

$$
f_{1}(p):=\left[\left(g\left(t_{1}, x\right)-\ell\right) \wedge w_{\mathrm{EU}}^{\sharp}\left(t_{1}, x, p\right)\right] \mathbf{1}_{\{p<\hat{p}\}}+\left[w_{\mathrm{EU}}^{\sharp}\left(t_{1}, x, p\right)-\ell\right] \mathbf{1}_{\{p \geqslant \hat{p}\}} .
$$

1. Proof of (1)(a) and (2)(a).

1.a First of all observe that $\tilde{p}>p_{\min }^{2}$ implies that $\tilde{g}\left(t_{1}, x, \ell\right)>0$. Moreover the definition of the closed convex envelope in both identities follows from Rockafellar [20, Theorem 12.2]. Then we define $\varphi: p \in \mathbb{R} \mapsto a\left[p-p_{\min }^{2}\right]^{+}$. We observe that in (1) $\varphi \leqslant f$ on $(-\infty, 1]$ and we can easily check that any candidate for the convex envelope of $f$ on its domain of definition is below $\varphi$.

1.b Similarly we define $\hat{\varphi}: p \in \mathbb{R} \mapsto \hat{a}\left[p-p_{\min }^{2}\right]^{+}$. As above we observe that in (2) $\hat{\varphi} \leqslant f$ on $(-\infty, \hat{p}]$. Then we set $k: p \in \mathbb{R} \mapsto \hat{\varphi}(p) \vee\left(w_{\mathrm{EU}}^{\sharp}\left(t_{1}, x, p\right)-\ell\right)$, which is convex. By definition, we have $w_{\mathrm{EU}}^{\sharp}\left(t_{1}, x, p\right)=0$ for $p \leqslant p_{\text {min }}^{2}$ (see Remark 4.5). Since $w_{\mathrm{EU}}^{\sharp}\left(t_{1}, x, p_{\text {min }}^{2}\right)=0$ and $w_{\mathrm{EU}}^{\sharp}\left(t_{1}, x, \hat{p}\right)=g\left(t_{1}, x\right)$, we have by convexity that $\left(w_{\mathrm{EU}}^{\sharp}\left(t_{1}, x, p\right)-\ell\right) \leqslant \hat{\varphi}(p)$ for $p \in[0, \hat{p}]$, and thus $k(p) \mathbf{1}_{\{p \leqslant \hat{p}\}}=\hat{\varphi}(p) \mathbf{1}_{\{p \leqslant \hat{p}\}}$. Besides we compute

$$
\mathrm{D}_{p}^{-} w_{\mathrm{EU}}^{\sharp}\left(t_{1}, x, \hat{p}\right) \geqslant \hat{a}>0 .
$$

Moreover thanks to the convexity of $w_{\mathrm{EU}}^{\sharp}(t, x, \cdot)$ and after recalling (4.11), we obtain that for $p \geqslant \hat{p}$,

$w_{\mathrm{EU}}^{\sharp}\left(t_{1}, x, p\right) \geqslant w_{\mathrm{EU}}^{\sharp}\left(t_{1}, x, \hat{p}\right)+\mathrm{D}_{p}^{-} w_{\mathrm{EU}}^{\sharp}\left(t_{1}, x, \hat{p}\right)(p-\hat{p}) \geqslant g\left(t_{1}, x\right)+\hat{a}(p-\hat{p})=\hat{\varphi}(p)+\ell$.

Hence $k(p) \mathbf{1}_{\{p \geqslant \hat{p}\}}=\left(w_{\mathrm{EU}}^{\sharp}\left(t_{1}, x, p\right)-\ell\right) \mathbf{1}_{\{p \geqslant \hat{p}\}}$. We observe that $k \leqslant f$. We can easily check that any candidate for the convex envelope of $f$ is below $k$. The property in (4.11) also shows that, in particular, for $p \geqslant \hat{p}, \mathrm{D}_{p}^{+} f(p)=\mathrm{D}_{p}^{+} w_{\mathrm{EU}}^{\sharp}\left(t_{1}, x, p\right)>0$ as $\hat{a}>0$.

2. Proof of (1)(b) and (2)(b).

In (1) $f^{\sharp}(q), q \geqslant 0$ is easily computed from $f^{\sharp \sharp}(p)$. Let us prove (2)(b). We observe 
that $w_{\mathrm{EU}}\left(t_{1}, x, q\right)<\infty$ for $q \geqslant 0$, since $w_{\mathrm{EU}}^{\sharp}\left(t_{1}, x, p\right)=\infty$ for $p>1$ (see Remark 4.5). Therefore the sub-differential of $w_{\mathrm{EU}}$ at non-negative $q$ is non empty. As in [2, proof of Lemma 3.1], the proof of (2)(b) is based on the subsequent results from convex analysis (see e.g. Ekeland and Teman [11, Chapter I Proposition 5.1]). We thus recall that $p$ is in the sub-differential of $\psi$ at $q$, where $\psi$ is a proper function on $\mathbb{R}$, if and only if

$$
\psi^{\sharp}(p)+\psi(q)=p q .
$$

2.a At $p=p_{\min }^{2}$, the sub-differential of $f^{\sharp \sharp}$ is equal to $[0, \hat{a}]$. This is a consequence of the characterization of the convex envelope of $f$ given in (2)(a). Appealing to the above equality with $\psi=f^{\sharp}$ and $(2)($ a) we then have for $q \in[0, \hat{a}]$,

$$
f^{\sharp \sharp}\left(p_{\text {min }}^{2}\right)+f^{\sharp}(q)=p_{\text {min }}^{2} q,
$$

leading to $f^{\sharp}(q)=p_{\min }^{2} q$ as $f^{\sharp \sharp}\left(p_{\text {min }}^{2}\right)=0$.

2.b The sub-differential of $f^{\sharp \sharp}$ at $\hat{p}$ is equal to $\mathcal{D}:=\left[\hat{a}, \mathrm{D}_{p}^{+} f(\hat{p})\right]$ if $\mathrm{D}_{p}^{+} f(\hat{p})<+\infty$ or $[\hat{a},+\infty)$ otherwise. This is again a consequence of (2)(a). Hence appealing to (4.12) with $\psi=f^{\sharp}$ and (2)(a) we have for $q \in \mathcal{D}$,

$$
f^{\sharp \sharp}(\hat{p})+f^{\sharp}(q)=\hat{p} q,
$$

leading to $f^{\sharp}(q)=\hat{p} q-\tilde{g}\left(t_{1}, x, \ell\right)$.

2.c If $q>\mathrm{D}_{p}^{+} f(\hat{p})$ then $p \geqslant \hat{p}$ as $q \in\left[\mathrm{D}_{p}^{-} f(p), \mathrm{D}_{p}^{+} f(p)\right]$ by [11, Chapter I Corollary 5.2]. On the other hand the sub-differential of $f$ is equal to the sub-differential of $w_{\mathrm{EU}}^{\sharp}\left(t_{1}, x, p\right)$ for $p \geqslant \hat{p}$. Therefore using (4.12) with $\psi=f^{\sharp}$ and (2)(a) we have for $q>\mathrm{D}_{p}^{+} f(\hat{p})$,

$$
f^{\sharp \sharp}(p)+f^{\sharp}(q)=w_{\mathrm{EU}}^{\sharp}\left(t_{1}, x, p\right)-\ell+f^{\sharp}(q)=p q,
$$

and as $\mathrm{D}_{p}^{+} f(p)=\mathrm{D}_{p}^{+} w_{\mathrm{EU}}^{\sharp}\left(t_{1}, x, p\right)$ for $p \geqslant \hat{p}$ and $w_{\mathrm{EU}}\left(t_{1}, x, \cdot\right)$ is convex, this leads to

$$
f^{\sharp}(q)=p q-w_{\mathrm{EU}}^{\sharp}\left(t_{1}, x, p\right)+\ell=w_{\mathrm{EU}}^{\sharp \sharp}\left(t_{1}, x, q\right)+\ell=w_{\mathrm{EU}}\left(t_{1}, x, q\right)+\ell .
$$

We now introduce some new sets that will be involved in the decomposition of $\left(w_{\mathrm{EU}}^{\sharp} \vee g\right)^{\sharp}$ and $\left(w_{\mathrm{EU}}^{\sharp} \vee g\right)^{\sharp \sharp}$ provided in the following proposition. We define:

(i) the subsets of $\mathcal{O}_{+}^{d} \times \mathbb{R}^{+}$,

$$
\begin{array}{ll}
A_{0}:=\left\{(x, \ell): \tilde{g}\left(t_{1}, x, \ell\right) \leqslant 0\right\}, & A_{1}^{1}:=\left\{(x, \ell): g\left(t_{1}, x\right)>0, \ell=0\right\}, \\
A_{1}^{2}:=\left\{(x, \ell): g\left(t_{1}, x\right)>0, \ell>0\right\}, & A_{2}^{1}:=\left\{(x, \ell): w_{\mathrm{EU}}^{\sharp}\left(t_{1}, x, 1\right) \leqslant \tilde{g}\left(t_{1}, x, \ell\right)\right\},
\end{array}
$$

(ii) the subsets of $\mathcal{O}_{+}^{d}$, 


$$
A_{2}^{2}:=\left\{x: w_{\mathrm{EU}}^{\sharp}\left(t_{1}, x, 1\right)>g\left(t_{1}, x\right)\right\} .
$$

From (i)-(ii) we derive the following subsets of $\mathcal{O}_{+}^{d} \times \mathbb{R}^{+}$,

$$
\begin{array}{ll}
A_{3}^{1}:=A_{1}^{2} \cap A_{2}^{1}, & A_{3}^{2}:=A_{1}^{2} \cap A_{0} \cap\left(\left(A_{2}^{2}\right)^{\mathrm{c}} \backslash A_{2}^{1}\right), \\
A_{3}^{3}:=A_{1}^{2} \cap A_{0}^{\mathrm{c}} \cap\left(\left(A_{2}^{2}\right)^{\mathrm{c}} \backslash A_{2}^{1}\right), & A_{3}^{4}:=A_{1}^{2} \cap A_{0} \cap A_{2}^{2}, \\
A_{3}^{5}:=A_{1}^{2} \cap A_{0}^{\mathrm{c}} \cap A_{2}^{2} . &
\end{array}
$$

Observe that $\left(A_{1}^{1} \cup A_{1}^{2}\right)^{\mathrm{c}}=\left\{x: g\left(t_{1}, x\right)=0\right\}$.

\section{Proposition 4.3.}

(a) For $x \in \mathcal{O}_{+}^{d}$, the function $\left(p_{1}, p_{2}\right) \mapsto\left(w_{\mathrm{EU}}^{\sharp} \vee g\right)^{\sharp \sharp}\left(t_{1}, x, p_{1}, p_{2}\right)=\overline{\operatorname{conv}}\left(w_{\mathrm{EU}}^{\sharp} \vee\right.$ $g)\left(t_{1}, x, p_{1}, p_{2}\right)$ is continuous on its domain and is given by (3.12) with $f \equiv w_{\mathrm{EU}}^{\sharp}$.

(b) On $\mathcal{O}_{+}^{d}$, and for all $\left(q_{1}, q_{2}\right) \in \mathbb{R}^{+} \times \mathbb{R}^{+}$,

$$
\begin{aligned}
\left(w_{\mathrm{EU}}^{\sharp} \vee g\right)^{\sharp}\left(t_{1}, \cdot, q_{1}, q_{2}\right)= & \tilde{w}_{\mathrm{EU}}\left(t_{1}, \cdot, q_{1}, q_{2}\right) \mathbf{1}_{\left(A_{1}^{1} \cup A_{1}^{2}\right)^{c}}(\cdot)+w_{\mathrm{EU}}\left(t_{1}, \cdot, q_{2}\right) \mathbf{1}_{A_{1}^{1} \cup A_{3}^{1}}\left(\cdot, q_{1}\right) \\
& +\left[q_{2}-\tilde{g}\left(t_{1}, \cdot, q_{1}\right)\right] \mathbf{1}_{A_{3}^{2}}\left(\cdot, q_{1}\right)+\left[p_{\min }^{2} q_{2} \vee\left(q_{2}-\tilde{g}\left(t_{1}, \cdot, q_{1}\right)\right)\right] \mathbf{1}_{A_{3}^{3}}\left(\cdot, q_{1}\right) \\
& +\kappa_{1}\left(t_{1}, \cdot, q_{1}, q_{2}\right) \mathbf{1}_{A_{3}^{4}}\left(\cdot, q_{1}\right)+\kappa_{2}\left(t_{1}, \cdot, q_{1}, q_{2}\right) \mathbf{1}_{A_{3}^{5}}\left(\cdot, q_{1}\right),
\end{aligned}
$$

where $\tilde{w}_{\mathrm{EU}}\left(t_{1}, \cdot, q_{1}, q_{2}\right):=w_{\mathrm{EU}}\left(t_{1}, \cdot, q_{2}\right)+q_{1}$ and

$\left\{\begin{array}{l}\kappa_{1}\left(t_{1}, \cdot, q_{1}, q_{2}\right):=\left[\hat{p} q_{2}-\tilde{g}\left(t_{1}, \cdot, q_{1}\right)\right] \mathbf{1}_{\left\{q_{2} \leqslant \bar{q}\left(t_{1}, \cdot \hat{p}\right)\right\}}+\tilde{w}_{\mathrm{EU}}\left(t_{1}, \cdot, q_{1}, q_{2}\right) \mathbf{1}_{\left\{q_{2}>\bar{q}\left(t_{1}, \cdot \hat{p}\right)\right\}} \\ \kappa_{2}\left(t_{1}, \cdot, q_{1}, q_{2}\right):=\left[p_{\min }^{2} q_{2} \vee\left(\hat{p} q_{2}-\tilde{g}\left(t_{1}, \cdot, q_{1}\right)\right)\right] \mathbf{1}_{\left\{q_{2} \leqslant \bar{q}\left(t_{1}, \cdot, \hat{p}\right)\right\}}+\tilde{w}_{\mathrm{EU}}\left(t_{1}, \cdot, q_{1}, q_{2}\right) \mathbf{1}_{\left\{q_{2}>\bar{q}\left(t_{1}, \cdot, \hat{p}\right)\right\}}\end{array}\right.$,

with $\bar{q}\left(t_{1}, \cdot, \hat{p}\right):=\mathrm{D}_{p}^{+} w_{\mathrm{EU}}^{\sharp}\left(t_{1}, \cdot, \hat{p}\right)$ and with $p_{\text {min }}^{2}, \tilde{g}, \hat{p}$, respectively defined in (4.2), (4.8) and (4.9).

Proof. We only prove (4.13). Thanks to the definition of the Legendre-Fenchel transform (recall the Notations) we have for $x \in \mathcal{O}_{+}^{d}$ and for all $\left(q_{1}, q_{2}\right) \in \mathbb{R}^{+} \times \mathbb{R}^{+}$,

$$
\begin{aligned}
\left(w_{\mathrm{EU}}^{\sharp} \vee g\right)^{\sharp}\left(t_{1}, x, q_{1}, q_{2}\right) & =\sup _{\left(p_{1}, p_{2}\right) \in \mathbb{R}^{2}}\left\{p_{1} q_{1}+p_{2} q_{2}-\left(w_{\mathrm{EU}}^{\sharp} \vee g\right)\left(t_{1}, x, p_{1}, p_{2}\right)\right\} \\
& =\sup _{p \leqslant 1}\left\{p q_{2}-f_{t_{1}, x, q_{1}}(p)\right\},
\end{aligned}
$$

with $f$ defined in Lemma 4.1, and where the last equality follows from Remark 4.6 and Remark 4.5. Now appealing to [11, Chapter I, Corollary 4.1] and the property $q_{2} \geqslant 0$, we obtain

$$
\left(w_{\mathrm{E} U}^{\sharp} \vee g\right)^{\sharp}\left(t_{1}, x, q_{1}, q_{2}\right)=\sup _{p \in[0,1]}\left\{p q_{2}-f_{t_{1}, x, q_{1}}^{\sharp \sharp}(p)\right\} .
$$


Using (4.10) and considering $q_{1} \geqslant 0$ we compute that on $\mathcal{O}_{+}^{d}$ for $p>1, f_{t_{1},,}^{\sharp \sharp} q_{1}(p)=\infty$, while for $p \leqslant 1$,

$$
\begin{aligned}
f_{t_{1}, \cdot, q_{1}}^{\sharp}(p):= & \tilde{w}_{\mathrm{EU}}^{\sharp}\left(t_{1}, \cdot, p, q_{1}\right) \mathbf{1}_{\left(A_{1}^{1} \cup A_{1}^{2}\right)}(\cdot) \\
& +w_{\mathrm{EU}}^{\sharp}\left(t_{1}, \cdot, p\right) \mathbf{1}_{A_{1}^{1} \cup A_{3}^{1}}\left(\cdot, q_{1}\right)+\tilde{g}\left(t_{1}, \cdot, q_{1}\right) \mathbf{1}_{A_{3}^{2}}\left(\cdot, q_{1}\right) \\
& +a\left[p-p_{\min }^{2}\right]^{+} \mathbf{1}_{A_{3}^{3}}\left(\cdot, q_{1}\right)+\kappa_{1}^{\sharp}\left(t_{1}, \cdot, p, q_{1}\right) \mathbf{1}_{A_{3}^{4}}\left(\cdot, q_{1}\right)+\kappa_{2}^{\sharp}\left(t_{1}, \cdot, p, q_{1}\right) \mathbf{1}_{A_{3}^{5}}\left(\cdot, q_{1}\right),
\end{aligned}
$$

where $\tilde{w}_{\mathrm{EU}}^{\sharp}\left(t_{1}, \cdot, p, q_{1}\right):=w_{\mathrm{EU}}^{\sharp}\left(t_{1}, \cdot, p\right)-q_{1}$ and

$$
\left\{\begin{array}{l}
\kappa_{1}^{\sharp}\left(t_{1}, \cdot, p, q_{1}\right):=\tilde{g}\left(t_{1}, \cdot, q_{1}\right) \mathbf{1}_{\{p \leqslant \hat{p}\}}+\tilde{w}_{\mathrm{EU}}^{\sharp}\left(t_{1}, \cdot, p, q_{1}\right) \mathbf{1}_{\{\hat{p}<p \leqslant 1\}} \\
\kappa_{2}^{\sharp}\left(t_{1}, \cdot, p, q_{1}\right):=\hat{a}\left[p-p_{\min }^{2}\right]^{+} \mathbf{1}_{\{p \leqslant \hat{p}\}}+\tilde{w}_{\mathrm{EU}}^{\sharp}\left(t_{1}, \cdot, p, q_{1}\right) \mathbf{1}_{\{\hat{p}<p \leqslant 1\}}
\end{array},\right.
$$

with $a$ and $\hat{a}$ defined in Lemma 4.1. The cases $A_{1}^{1} \cup A_{3}^{1},\left(A_{1}^{1} \cup A_{1}^{2}\right)^{\mathrm{c}}, A_{3}^{2}$ are straightforward. The cases $A_{3}^{3}$ and $A_{3}^{5}$ have been treated in Lemma 4.1 while the case $A_{3}^{4}$ can be studied similarly.

\section{Remark 4.7.}

(a) Proposition 4.3 implies that for $(t, x) \in\left[t_{0}, t_{1}\right) \times \mathcal{O}_{+}^{d}, w_{\mathrm{EU}}(t, x, \cdot)$ is a proper convex non-decreasing and non-negative function. Moreover, on $\left[t_{0}, t_{1}\right) \times \mathcal{O}_{+}^{d}, w_{\mathrm{EU}}(\cdot, 0,0)=0$ and $w_{\mathrm{EU}}\left(\cdot, q_{1}, q_{2}\right)=\infty$ for $\left\{q_{1}<0\right\} \cup\left\{q_{2}<0\right\}$.

(b) On $\mathcal{O}_{+}^{d}$, we have $\mathrm{D}_{p}^{+} w_{\mathrm{EU}}^{\sharp}\left(t_{1}, \cdot, \hat{p}\right)>0$ on $A_{3}^{4}$ and $A_{3}^{5}$, as $w_{\mathrm{EU}}^{\sharp}\left(t_{1}, \cdot, \hat{p}\right) \geqslant g\left(t_{1}, \cdot\right)>0$ and $w_{\mathrm{EU}}^{\sharp}\left(t_{1}, \cdot, 0\right)=0$ (see Remark 4.5). Moreover as $w_{\mathrm{EU}}^{\sharp}\left(t_{1}, \cdot, 1\right)=v_{\mathrm{BM}}\left(t_{1}, \cdot, 1\right)$ on $\mathcal{O}_{+}^{d}$ (see Remark 4.5) and $\hat{p}<1$ on both sets, we conclude that $\mathrm{D}_{p}^{+} w_{\mathrm{EU}}^{\sharp}\left(t_{1}, \cdot, \hat{p}\right)<+\infty$.

We can now turn to the study of the sub-differential of $w_{\mathrm{EU}}$. Recall the definition of $p_{\min }^{k}, 1 \leqslant k \leqslant 2$ in (4.2).

Lemma 4.2. Fix $(t, x) \in\left[t_{0}, t_{1}\right) \times \mathcal{O}_{+}^{d}$. Then for $q_{2} \in \mathbb{R}^{+}$:

(a) $\mathrm{D}_{q_{1}}^{+} w_{\mathrm{EU}}\left(t, x, q_{1}, q_{2}\right) \geqslant 0$ if $q_{1} \geqslant 0$, and $\mathrm{D}_{q_{1}}^{-} w_{\mathrm{EU}}\left(t, x, q_{1}, q_{2}\right) \geqslant 0$ if $q_{1}>0$,

(b) $\lim _{q_{1} \uparrow \infty} \mathrm{D}_{q_{1}}^{ \pm} w_{\mathrm{EU}}\left(t, x, q_{1}, q_{2}\right)=1$,

(c) $\mathrm{D}_{q_{1}}^{+} w_{\mathrm{EU}}\left(t, x, 0, q_{2}\right)=p_{\min }^{1}(t, x)$.

Moreover for $q_{1} \in \mathbb{R}^{+}$:

(d) $\mathrm{D}_{q_{2}}^{+} w_{\mathrm{EU}}\left(t, x, q_{1}, q_{2}\right) \geqslant 0$ if $q_{2} \geqslant 0$ and $\mathrm{D}_{q_{2}}^{-} w_{\mathrm{EU}}\left(t, x, q_{1}, q_{2}\right) \geqslant 0$ if $q_{2}>0$,

(e) $\lim _{q_{2} \uparrow \infty} \mathrm{D}_{q_{2}}^{ \pm} w_{\mathrm{EU}}\left(t, x, q_{1}, q_{2}\right)=1$.

Moreover

$$
\left\{\begin{array}{ll}
\mathrm{D}_{q_{1}}^{-} w_{\mathrm{EU}}\left(t, x, q_{1}, q_{2}\right)=\mathbb{E}\left[\mathrm{D}_{q_{1}}^{-}\left(w_{\mathrm{EU}}^{\sharp} \vee g\right)^{\sharp}\left(t_{1}, X_{t_{1}}^{t, x}, q_{1} Q_{t_{1}}^{t, x, 1}, q_{2} Q_{t_{1}}^{t, x, 1}\right)\right] & \text { for } q_{1}>0, q_{2} \geqslant 0 \\
\mathrm{D}_{q_{2}}^{-} w_{\mathrm{EU}}\left(t, x, q_{1}, q_{2}\right)=\mathbb{E}\left[\mathrm{D}_{q_{2}}^{-}\left(w_{\mathrm{EU}}^{\sharp} \vee g\right)^{\sharp}\left(t_{1}, X_{t_{1}}^{t, x}, q_{1} Q_{t_{1}}^{t, x, 1}, q_{2} Q_{t_{1}}^{t, x, 1}\right)\right] & \text { for } q_{1} \geqslant 0, q_{2}>0 \\
\mathrm{D}_{q_{k}}^{+} w_{\mathrm{EU}}\left(t, x, q_{1}, q_{2}\right)=\mathbb{E}\left[\mathrm{D}_{q_{k}}^{+}\left(w_{\mathrm{EU}}^{\sharp} \vee g\right)^{\sharp}\left(t_{1}, X_{t_{1}}^{t, x}, q_{1} Q_{t_{1}}^{t, x, 1}, q_{2} Q_{t_{1}}^{t, x, 1}\right)\right] & \text { for } q_{1}, q_{2} \geqslant 0, k \in\{1,2\}
\end{array} .\right.
$$


Proof. This is a direct consequence of (4.2), (4.7), Proposition 4.3, Remark 4.5, Remark 4.7 (b).

Remark 4.8. Note that on $\left[t_{0}, t_{1}\right) \times \mathcal{O}_{+}^{d}, \mathrm{D}_{q_{1}}^{-} w_{\mathrm{EU}}\left(\cdot, 0, q_{2}\right)\left(\right.$ resp. $\left.\mathrm{D}_{q_{2}}^{-} w_{\mathrm{EU}}\left(\cdot, q_{1}, 0\right)\right), q_{2} \geqslant 0$ $\left(\right.$ resp. $\left.q_{1} \geqslant 0\right)$ is $-\infty$ as $w_{\mathrm{EU}}\left(\cdot, q_{1}, q_{2}\right)=\infty$ for $q_{1}<0$ (resp. $\left.q_{2}<0\right)$ (see (4.7)).

\subsubsection{The backward algorithm as an upper-bound}

We can now work towards the proof of the dual algorithm as an upper bound to $v_{\mathrm{EU}}$. However we prove three intermediary results before reaching the final argument.

Bounds and limits for $w_{\mathrm{EU}}^{\sharp}$ Our first intermediary result is related to the behavior of $w_{\mathrm{EU}}^{\sharp}$ when $\left(p_{1}, p_{2}\right)=(1,1)$. This result will be involved in the proof of Theorem 4.2.

Lemma 4.3. Fix $(t, x) \in\left[t_{0}, t_{1}\right) \times \mathcal{O}_{+}^{d}$. Then $w_{\mathrm{EU}}^{\sharp}(t, x, \cdot)$ is proper, convex, non-negative, non-decreasing on its domain $(-\infty, 1] \times(-\infty, 1]$ and

$$
0 \leqslant w_{\mathrm{EU}}^{\sharp}(t, x, \cdot) \leqslant w_{\mathrm{EU}}^{\sharp}(t, x, 1,1)=v_{\mathrm{BM}}(t, x, 1) \text { on }(-\infty, 1] \times(-\infty, 1] \text {, }
$$

with $v_{\mathrm{BM}}(t, x, 1)$ defined in $(3.15)$.

Proof. We consider $(t, x) \in\left[t_{0}, t_{1}\right) \times \mathcal{O}_{+}^{d}$. We know from [20, Theorem 12.2] that $w_{\mathrm{EU}}^{\sharp}(t, x, \cdot)$ is closed, convex, proper, non-decreasing, non-negative (see Remark 4.7 (a)) on its domain and thus continuous on the interior of its domain. We now notice that (4.7) implies for $\left(q_{1}, q_{2}\right) \in(0, \infty)^{2}$,

$$
\begin{aligned}
\delta\left(q_{1}, q_{2}\right) & :=q_{1}+q_{2}-w_{\mathrm{EU}}\left(t, x, q_{1}, q_{2}\right) \\
& =\mathbb{E}^{\mathbb{Q} t, x}\left[\left(q_{1}+q_{2}\right) Q_{t_{1}}^{t, x, 1}-\left(w_{\mathrm{EU}}^{\sharp} \vee g\right)^{\sharp}\left(t_{1}, X_{t_{1}}^{t, x}, q_{1} Q_{t_{1}}^{t, x, 1}, q_{2} Q_{t_{1}}^{t, x, 1}\right)\right],
\end{aligned}
$$

where $\left(q_{1}, q_{2}\right) \mapsto \delta\left(q_{1}, q_{2}\right)$ is non-decreasing by Lemma 4.2. Applying the Monotone Convergence Theorem and using Proposition 4.3 and Remark 4.5 we get

$$
\lim _{\substack{q_{1} \rightarrow \infty \\ q_{2} \rightarrow \infty}} \delta\left(q_{1}, q_{2}\right)=\mathbb{E}^{\mathbb{Q}_{t, x}}\left[\left(v_{\mathrm{EU}} \vee g\right)\left(t_{1}, X_{t_{1}}^{t, x}, 1,1\right)\right]=v_{\mathrm{EU}}(t, x, 1,1)=v_{\mathrm{BM}}(t, x, 1),
$$

(recall Proposition 4.2 and the definition of the admissible set of controls). This leads to

$$
w_{\mathrm{EU}}^{\sharp}(t, x, 1,1)=\sup _{\left(q_{1}, q_{2}\right) \in(0, \infty)^{2}} \delta\left(q_{1}, q_{2}\right) \geqslant \lim _{q_{1} \rightarrow \infty, q_{2} \rightarrow \infty} \delta\left(q_{1}, q_{2}\right)=v_{\mathrm{EU}}(t, x, 1,1) .
$$

We conclude noticing that we already have $w_{\mathrm{EU}}^{\sharp}(t, x, 1,1) \leqslant v_{\mathrm{EU}}(t, x, 1,1)$ (recall the discussion at the beginning of Section 4.3). 
Moreover if $p_{2} \in(-\infty, 1], p_{1}>1$ then

$$
w_{\mathrm{EU}}^{\sharp}\left(t, x, p_{1}, p_{2}\right) \geqslant \lim _{q_{1} \rightarrow \infty}\left\{p_{1} q_{1}-w_{\mathrm{EU}}\left(t, x, q_{1}, 0\right)\right\}=\infty,
$$

as $\lim _{q_{1} \rightarrow \infty} \mathrm{D}_{q_{1}}^{+} w_{\mathrm{EU}}\left(t, x, q_{1}, 0\right)=1$ (see Lemma $4.2(\mathrm{~b})$ ). Similar results are obtained when $p_{1} \in(-\infty, 1], p_{2}>1$.

Finally we know from Remark 4.7 (a) that on $\left[t_{0}, t_{1}\right) \times \mathcal{O}_{+}^{d}, w_{\mathrm{EU}}^{\sharp}\left(\cdot, p_{1}, p_{2}\right)=0$ for $\left(p_{1}, p_{2}\right) \in(-\infty, 0] \times(-\infty, 0]$.

Therefore the non-decreasing property of $w_{\mathrm{EU}}^{\sharp}(t, x, \cdot)$ combined with the latter results lead to the conclusion that the domain of $w_{\mathrm{EU}}^{\sharp}(t, x, \cdot)$ is actually $(-\infty, 1] \times(-\infty, 1]$.

Convexification in the dynamic programming algorithm As mentioned in Remark 4.4, we will prove that $v_{\mathrm{EU}} \vee g$ can be replaced by its convex envelope, with respect to $\left(p_{1}, p_{2}\right)$ in (4.5). Later, we will check in Theorem 4.2 that a similar result holds for $w_{\mathrm{EU}}^{\sharp}$. Observe that the two identities (4.15) and (4.18) below imply that $v_{\mathrm{EU}}=w_{\mathrm{EU}}^{\sharp}$ on $\left[t_{0}, t_{1}\right) \times \mathcal{O}_{+}^{d} \times[0,1]^{2}$ since we already have $v_{\mathrm{EU}} \geqslant w_{\mathrm{EU}}^{\sharp}$.

Proposition 4.4. For all $t \in\left[t_{0}, t_{1}\right)$ and $\left(x, p_{1}, p_{2}\right) \in \mathcal{O}_{+}^{d} \times[0,1]^{2}$, we have

$$
v_{\mathrm{EU}}\left(t, x, p_{1}, p_{2}\right)=\inf _{\left(\alpha_{1}, \alpha_{2}\right) \in \mathcal{A}_{t, p_{1}}^{t_{1}} \times \mathcal{A}_{t, p_{2}}^{t_{2}}} \mathbb{E}^{\mathbb{Q} t, x}\left[\overline{\operatorname{conv}}\left(v_{\mathrm{EU}} \vee g\right)\left(t_{1}, X_{t_{1}}^{t, x}, P_{t_{1}}^{t, p_{1}, \alpha_{1}}, P_{t_{1}}^{t, p_{2}, \alpha_{2}}\right)\right],
$$

where $\overline{\operatorname{conv}}\left(v_{\mathrm{EU}} \vee g\right)$ is the closed convex envelope of $\left(v_{\mathrm{EU}} \vee g\right)$ (with respect to $\left(p_{1}, p_{2}\right)$ ) given by (3.12) with $f \equiv v_{\mathrm{EU}}$.

Proof. We fix $(t, x) \in\left[t_{0}, t_{1}\right) \times \mathcal{O}_{+}^{d}$. If $p_{1}=\{0,1\}$ then $\mathcal{A}_{t, p_{1}}^{t_{1}}=\{0\}$ and (4.15) holds for any $p_{2} \in[0,1]$ thanks to the explicit definition of $\overline{\operatorname{conv}}\left(v_{\mathrm{EU}} \vee g\right)\left(t_{1}, x, p_{1}, p_{2}\right)$, the convexity of $v_{\mathrm{EU}}\left(t_{1}, x, \cdot\right)$ (recall that we already have $v_{\mathrm{EU}}\left(t_{1}, \cdot\right)=w_{\mathrm{EU}}^{\sharp}\left(t_{1}, \cdot\right)$ on their domain) and (4.5). In particular, we can check that when $\left(p_{1}, p_{2}\right)=(0,0)\left(\operatorname{resp} .\left(p_{1}, p_{2}\right)=(1,1)\right)$, $v_{\mathrm{EU}}\left(t, x, p_{1}, p_{2}\right)=0\left(\operatorname{resp} . v_{\mathrm{EU}}\left(t, x, p_{1}, p_{2}\right)=v_{\mathrm{BM}}(t, x, 1)\right.$, with $v_{\mathrm{BM}}(t, x, 1)$ defined in $(3.15))$ as $\mathcal{A}_{t, p_{1}}^{t_{1}}=\{0\}$ and $\mathcal{A}_{t, p_{2}}^{t_{2}}=\{0\}$.

We must show (4.15) for $p_{2} \in[0,1], 0<p_{1}<1$. Considering Proposition 4.2 this amounts to proving that

$$
\begin{aligned}
\inf _{\left(\alpha_{1}, \alpha_{2}\right) \in \mathcal{A}_{t, p_{1}}^{t_{1}} \times \mathcal{A}_{t, p_{2}}^{t_{2}}} \mathbb{E}^{\mathbb{Q} t, x}\left[\overline{\operatorname{conv}}\left(v_{\mathrm{EU}} \vee g\right)\left(t_{1}, X_{t_{1}}^{t, x}, P_{t_{1}}^{t, p_{1}, \alpha_{1}}, P_{t_{1}}^{t, p_{2}, \alpha_{2}}\right)\right] & \\
\geqslant & \inf _{\left(\alpha_{1}, \alpha_{2}\right) \in \mathcal{A}_{t, p_{1}}^{t_{1}} \times \mathcal{A}_{t, p_{2}}^{t_{2}}} \mathbb{E}^{\mathbb{Q}_{t, x}}\left[\left(v_{\mathrm{EU}} \vee g\right)\left(t_{1}, X_{t_{1}}^{t, x}, P_{t_{1}}^{t, p_{1}, \alpha_{1}}, P_{t_{1}}^{t, p_{2}, \alpha_{2}}\right)\right],
\end{aligned}
$$


the reverse inequality being satisfied by definition. Moreover it follows from the explicit definition of $\overline{\operatorname{conv}}\left(v_{\mathrm{EU}} \vee g\right)\left(t_{1}, x, p_{1}, p_{2}\right)$ and the convexity of $v_{\mathrm{EU}}\left(t_{1}, x, \cdot\right)$ that the closed convex envelope in $p_{1}$ is actually the closed convex envelope in $\left(p_{1}, p_{2}\right)$. As a consequence, to prove (4.16), we just need to build the closed convex envelope with respect to $p_{1}$, by arguing as in [2, proof of Proposition 3.4].

More precisely, we know from the Carathéodory theorem that we can find two maps $\left(\lambda_{j}, \pi_{j}\right):\left(x, p_{1}, p_{2}\right) \in \mathcal{O}_{+}^{d} \times[0,1]^{2} \mapsto\left(\lambda_{j}, \pi_{j}\right)\left(x, p_{1}, p_{2}\right) \in[0,1] \times[0,1], j \leqslant 2$, such that

$$
\sum_{j=1}^{2} \pi_{j}\left(x, p_{1}, p_{2}\right)=1, p_{1}=\sum_{j=1}^{2} \pi_{j}\left(x, p_{1}, p_{2}\right) \lambda_{j}\left(x, p_{1}, p_{2}\right)
$$

and $\overline{\mathrm{conv}}\left(v_{\mathrm{EU}} \vee g\right)\left(t_{1}, x, p_{1}, p_{2}\right)=\sum_{j=1}^{2} \pi_{j}\left(x, p_{1}, p_{2}\right)\left(v_{\mathrm{EU}} \vee g\right)\left(t_{1}, x, \lambda_{j}\left(x, p_{1}, p_{2}\right), p_{2}\right)$.

They can be chosen in a measurable way. Indeed we first notice the continuity of $v_{\mathrm{EU}}\left(t_{1}, \cdot\right)$ on $\mathcal{O}_{+}^{d} \times[0,1]$ (see Remark 4.5) and thus of $\overline{\operatorname{conv}}\left(v_{\mathrm{EU}} \vee g\right)\left(t_{1}, \cdot\right)$ on $\mathcal{O}_{+}^{d} \times$ $[0,1] \times[0,1]$ (recall $(3.9)$ and $(3.12))$. We then appeal to Bertsekas and Shreve [1, Proposition 7.49$]$ to choose $\left(\lambda_{j}, \pi_{j}\right)$ to be analytically measurable. We finally use [1, Lemma 7.27] to obtain a Borel-measurable version which coincides a.e. for the pull-back measure of $\left(X_{t_{1}-\varepsilon}^{t, x}, P_{t_{1}-\varepsilon}^{t, p_{1}, \alpha_{1}}, P_{t_{1}-\varepsilon}^{t, p_{2}, \alpha_{2}}\right)$, with $\alpha_{1} \in \mathcal{A}_{t, p_{1}}^{t_{1}}, \alpha_{2} \in \mathcal{A}_{t, p_{2}}^{t_{2}}$ and $0<\varepsilon<t_{1}-t$ fixed. We use this version in what follows.

We now introduce $\xi$, an $\mathcal{F}_{t_{1}}$-measurable random variable being such that

$$
\mathbb{P}\left[\xi=\lambda_{j}\left(X_{t_{1}-\varepsilon}^{t, x}, P_{t_{1}-\varepsilon}^{t, p_{1}, \alpha_{1}}, P_{t_{1}-\varepsilon}^{t, p_{2}, \alpha_{2}}\right) \mid \mathcal{F}_{t_{1}-\varepsilon}\right]=\pi_{j}\left(X_{t_{1}-\varepsilon}^{t, x}, P_{t_{1}-\varepsilon}^{t, p_{1}, \alpha_{1}}, P_{t_{1}-\varepsilon}^{t, p_{2}, \alpha_{2}}\right) .
$$

The above construction implies that $\mathbb{E}\left[\xi \mid \mathcal{F}_{t_{1}-\varepsilon}\right]=P_{t_{1}-\varepsilon}^{t, p_{1}, \alpha_{1}}$. We can thus find $\alpha_{1, \varepsilon} \in \mathcal{A}_{t, p_{1}}^{t_{1}}$ such that $P_{t_{1}-\varepsilon}^{t, p_{1}, \alpha_{1, \varepsilon}}=P_{t_{1}-\varepsilon}^{t, p_{1}, \alpha_{1}}$ and $P_{t_{1}}^{t, p_{1}, \alpha_{1, \varepsilon}}=\xi$. Then using (4.17) we obtain, after denoting $Q_{s}:=Q_{s}^{t, x, 1}, t \leqslant s \leqslant T$,

$$
\begin{aligned}
\mathbb{E}\left[Q_{t_{1}-\varepsilon}^{-1} \times\right. & \left.\overline{\operatorname{conv}}\left(v_{\mathrm{EU}} \vee g\right)\left(t_{1}, X_{t_{1}-\varepsilon}^{t, x}, P_{t_{1}-\varepsilon}^{t, p_{1}, \alpha_{1}}, P_{t_{1}-\varepsilon}^{t, p_{2}, \alpha_{2}}\right)\right] \\
= & \mathbb{E}^{\mathbb{Q} t, x}\left[\left(v_{\mathrm{EU}} \vee g\right)\left(t_{1}, X_{t_{1}-\varepsilon}^{t, x}, P_{t_{1}}^{t, p_{1}, \alpha_{1, \varepsilon}}, P_{t_{1}-\varepsilon}^{t, p_{2}, \alpha_{2}}\right)\right] \\
& -\mathbb{E}\left[\left(Q_{t_{1}}^{-1}-Q_{t_{1}-\varepsilon}^{-1}\right)\left(v_{\mathrm{EU}} \vee g\right)\left(t_{1}, X_{t_{1}-\varepsilon}^{t, x}, P_{t_{1}}^{t, p_{1}, \alpha_{1, \varepsilon}}, P_{t_{1}-\varepsilon}^{t, p_{2}, \alpha_{2}}\right)\right] .
\end{aligned}
$$

Recalling (3.8)-(3.9), (4.4) and (4.6), we thus conclude

$$
\begin{aligned}
\mathbb{E}\left[Q_{t_{1}-\varepsilon}^{-1} \overline{\operatorname{conv}}\left(v_{\mathrm{EU}} \vee g\right)\left(t_{1}, X_{t_{1}-\varepsilon}^{t, x}, P_{t_{1}-\varepsilon}^{t, p_{1}, \alpha_{1}}, P_{t_{1}-\varepsilon}^{t, p_{2}, \alpha_{2}}\right)\right] \\
\geqslant \inf _{\alpha_{1} \in \mathcal{A}_{t, p_{1}}^{t_{1}}} \mathbb{E}^{\mathbb{Q} t, x}\left[\left(v_{\mathrm{EU}} \vee g\right)\left(t_{1}, X_{t_{1}}^{t, x}, P_{t_{1}}^{t, p_{1}, \alpha_{1}}, P_{t_{1}}^{t, p_{2}, \alpha_{2}}\right)\right]+\Delta(\varepsilon),
\end{aligned}
$$


with

$$
\begin{aligned}
\Delta(\varepsilon)= & -C \mathbb{E}^{\mathbb{Q}_{t, x}}\left[\left|X_{t_{1}-\varepsilon}^{t, x}-X_{t_{1}}^{t, x}\right|\left(1+\left|X_{t_{1}-\varepsilon}^{t, x}\right|+\left|X_{t_{1}}^{t, x}\right|\right)\right] \\
& -C \mathbb{E}^{\mathbb{Q}_{t, x}}\left[\left|v_{\mathrm{EU}}\left(t_{1}, X_{t_{1}}^{t, x}, P_{t_{1}-\varepsilon}^{t, p_{2}, \alpha_{2}}\right)-v_{\mathrm{EU}}\left(t_{1}, X_{t_{1}}^{t, x}, P_{t_{1}}^{t, p_{2}, \alpha_{2}}\right)\right|\right] \\
& -C \mathbb{E}^{\mathbb{Q}_{t, x}}\left[\left|1-Q_{t_{1}} / Q_{t_{1}-\varepsilon}\right|\left(1+\left|X_{t_{1}-\varepsilon}^{t, x}\right|\right)\right],
\end{aligned}
$$

for some $C>0$. Moreover since (3.8) and (4.4) hold, we have $0 \leqslant \overline{\operatorname{conv}}\left(v_{\mathrm{EU}} \vee\right.$ $g)\left(t_{1}, x, \cdot\right) \leqslant v_{\mathrm{EU}} \vee g\left(t_{1}, x, \cdot\right) \leqslant C(1+|x|)$. We can then use the continuity of both $v_{\mathrm{EU}}\left(t_{1}, x, \cdot\right)$ on $[0,1]\left(\right.$ recall Remark 4.5) and $\overline{\operatorname{conv}}\left(v_{\mathrm{EU}} \vee g\right)\left(t_{1}, \cdot\right)$ on $\mathcal{O}_{+}^{d} \times[0,1] \times[0,1]$ to pass to the limit in order to obtain, with (3.2)-(3.3) and (3.5), (4.16).

Since our final result is $v_{\mathrm{EU}}=w_{\mathrm{EU}}^{\sharp}$ we expect the same convexification in the dual algorithm. This is what we intend to prove in the following theorem. However the proof of the latter theorem requires, at some point, to find a particular value $\left(p_{1}, p_{2}\right)$ in the sub-differential of $w_{\mathrm{EU}}$. This is possible if $\mathrm{D}_{q_{1}}^{ \pm} w, \mathrm{D}_{q_{2}}^{ \pm} w_{\mathrm{EU}}$ are regular enough. To ensure this we make the following assumption which will be related to the regularity of $\mathrm{D}_{q_{1}}^{+} w_{\mathrm{EU}}$. No additional assumptions are needed for $\mathrm{D}_{q_{1}}^{-} w, \mathrm{D}_{q_{2}}^{ \pm} w_{\mathrm{EU}}$.

Assumption 4.3.1. For all $(t, x) \in[0, T] \times \mathcal{O}_{+}^{d}$, we have

$$
\mathbb{P}\left[q Q_{t_{1}}^{t, x, 1}=g\left(t_{1}, X_{t_{1}}^{t, x}\right)-v_{\mathrm{EU}}\left(t_{1}, X_{t_{1}}^{t, x}, 1\right)\right]=0 \forall q>0 .
$$

Remark 4.9.

(a) Assumption 4.3.1 holds for $w_{\mathrm{EU}}^{\sharp}$ in place of $v_{E U}$ by Remark 4.5.

(b) Assumption 4.3.1 holds, for instance, when $g$ is the payoff function of a put option since in this case $v_{E U}\left(t_{1}, \cdot, 1\right) \geqslant g\left(t_{1}, \cdot\right)$ on $\mathcal{O}_{+}^{d}$.

Theorem 4.2. Let Assumption 4.3.1 hold and fix $(t, x) \in\left[t_{0}, t_{1}\right) \times \mathcal{O}_{+}^{d}$. Then, for $\left(p_{1}, p_{2}\right) \in[0,1]^{2}$, there exists $\left(\hat{\alpha}_{1}, \hat{\alpha}_{2}\right) \in \mathcal{A}_{t, p_{1}}^{t_{1}} \times \mathcal{A}_{t, p_{2}}^{t_{2}}$ such that

$$
w_{\mathrm{EU}}^{\sharp}\left(t, x, p_{1}, p_{2}\right)=\mathbb{E}^{\mathbb{Q} t, x}\left[\overline{\operatorname{conv}}\left(w_{\mathrm{EU}}^{\sharp} \vee g\right)\left(t_{1}, X_{t_{1}}^{t, x}, P_{t_{1}}^{t, p_{1}, \hat{\alpha}_{1}}, P_{t_{1}}^{t, p_{2}, \hat{\alpha}_{2}}\right)\right],
$$

where $\overline{\mathrm{conv}}\left(w_{\mathrm{EU}}^{\sharp} \vee g\right)$ is the closed convex envelope of $\left(w_{\mathrm{EU}}^{\sharp} \vee g\right)$ (with respect to $\left(p_{1}, p_{2}\right)$ ) given by (3.12) with $f \equiv w_{\mathrm{EU}}^{\sharp}$.

In particular $w_{\mathrm{EU}}^{\sharp}(t, x, \cdot)$ is continuous on its domain.

Proof. Observe that the continuity of $w_{\mathrm{EU}}^{\sharp}$ is a direct consequence of Remark 4.5 and (3.12). We now prove (4.18). We recall the definition of $p_{\min }^{k}, k \in\{1,2\}$ in (4.2). For $\left(t, x, q_{1}, q_{2}\right) \in\left[t_{0}, t_{1}\right) \times \mathcal{O}_{+}^{d} \times \mathbb{R}^{+^{2}},(\lambda, \beta) \in[0,1] \times\{0,1\}$, and for $k \in\{1,2\}$, we define on $\left[t, t_{1}\right)$ the process

$Z^{t, x, q_{1}, q_{2}, k}(\lambda, \beta):=\left(\lambda \mathrm{D}_{q_{k}}^{+}\left(w_{\mathrm{EU}}^{\sharp} \vee g\right)^{\sharp}+\beta(1-\lambda) \mathrm{D}_{q_{k}}^{-}\left(w_{\mathrm{EU}}^{\sharp} \vee g\right)^{\sharp}\right)\left(\cdot, X^{t, x}, q_{1} Q^{t, x, 1}, q_{2} Q^{t, x, 1}\right)$. 
We fix $(t, x) \in\left[t_{0}, t_{1}\right) \times \mathcal{O}_{+}^{d}$. From now on we omit the dependency in $(t, x)$ of $Z^{t, x, q_{1}, q_{2}, k}(\lambda, \beta)$ and we will not write all the dependencies of the other variables involved in the subsequent discussion.

1. We assume that $\left(p_{1}, p_{2}\right) \in[0,1)^{2}$. We consider, for $\left(q_{k}, \lambda_{k}, \beta_{k}\right)_{k \in\{1,2\}} \in \mathbb{R}^{+} \times[0,1] \times$ $\{0,1\}$, the system

$$
\left\{\begin{array}{l}
p_{1}=\mathbb{E}\left[Z_{t_{1}}^{q_{1}, q_{2}, 1}\left(\lambda_{1}, \beta_{1}\right)\right] \\
p_{2}=\mathbb{E}\left[Z_{t_{1}}^{q_{1}, q_{2}, 2}\left(\lambda_{2}, \beta_{2}\right)\right]
\end{array} .\right.
$$

1.a Proof of a solution $\left(\hat{q}_{1}, \lambda_{1}, \beta_{1}\right)$ and $\left(\hat{q}_{2}, \lambda_{2}, \beta_{2}\right)$ to (4.19).

1.a.1 For each $q_{2} \geqslant 0$, assume that $p_{1} \in\left(p_{\min }^{1}(t, x), 1\right)$ (resp. $p_{1} \in\left[0, p_{\min }^{1}(t, x)\right]$ ). It follows from Lemma 4.2 (a)-(c) and Assumption 4.3.1 that there exists a $\hat{q}_{1}\left(q_{2}\right) \in$ $(0, \infty)$ (resp. $\hat{q}_{1}\left(q_{2}\right)=0$, recall Remark 4.8) such that $p_{1}$ lies in the sub-differential of $w_{\mathrm{EU}}\left(t, x, \cdot, q_{2}\right)$ at $\hat{q}_{1}\left(q_{2}\right)$. Therefore we can find $\left(\lambda_{1}, \beta_{1}\right) \in[0,1] \times\{1\}$ (see $\left.(4.14)\right)$ such that

$$
p_{1}=\mathbb{E}\left[Z_{t_{1}}^{\hat{q}_{1}\left(q_{2}\right), q_{2}, 1}\left(\lambda_{1}, \beta_{1}\right)\right],
$$

(resp. $\left(\lambda_{1}, \beta_{1}\right) \in[0,1] \times\{0\}$ such that (4.20) holds).

1.a.2 Now assume that $p_{2} \in\left(\mathrm{D}_{q_{2}}^{+} w_{\mathrm{EU}}\left(t, x, \hat{q}_{1}(0), 0\right), 1\right)\left(\operatorname{resp} .\left[0, \mathrm{D}_{q_{2}}^{+} w_{\mathrm{EU}}\left(t, x, \hat{q}_{1}(0), 0\right)\right]\right)$, with $\hat{q}_{1}\left(q_{2}\right), q_{2} \geqslant 0$ defined in the previous step. It follows from Lemma $4.2(\mathrm{~d})-(\mathrm{e})$ that there exists a $\hat{q}_{2} \in(0, \infty)$ (resp. $\hat{q}_{2}=0$, recall Remark 4.8) such that $p_{2}$ lies in the sub-differential of $w_{\mathrm{EU}}\left(t, x, \hat{q}_{1}\left(\hat{q}_{2}\right), \cdot\right)$ at $\hat{q}_{2}$. Therefore we can find $\left(\lambda_{2}, \beta_{2}\right) \in[0,1] \times\{1\}$ (see (4.14)) such that

$$
p_{2}=\mathbb{E}\left[Z_{t_{1}}^{\hat{q}_{1}\left(\hat{q}_{2}\right), \hat{q}_{2}, 2}\left(\lambda_{2}, \beta_{2}\right)\right],
$$

(resp. $\left(\lambda_{2}, \beta_{2}\right) \in[0,1] \times\{0\}$ such that (4.21) holds).

1.b Characterization of $\hat{\alpha}$.

We know from Lemma 4.2 that the random variable in both expectations in (4.20)(4.21) is valued in $[0,1]$. By the Martingale Representation Theorem we can thus find $\left(\hat{\alpha}_{1}, \hat{\alpha}_{2}\right) \in \mathcal{A}_{t, p_{1}}^{t_{1}} \times \mathcal{A}_{t, p_{2}}^{t_{1}}$ such that

$$
\left\{\begin{array}{l}
Z_{t_{1}\left(\hat{q}_{2}\right), \hat{q}_{2}, 1}^{\hat{q}_{1}}\left(\lambda_{1}, \beta_{1}\right)=p_{1}+\int_{t}^{t_{1}} \hat{\alpha}_{1, s}^{\top} \mathrm{d} W_{s}=: P_{t_{1}}^{t, p_{1}, \hat{\alpha}_{1}} \\
Z_{t_{1}}^{\hat{q}_{1}\left(\hat{q}_{2}\right), \hat{q}_{2}, 2}\left(\lambda_{2}, \beta_{2}\right)=p_{2}+\int_{t}^{t_{1}} \hat{\alpha}_{2, s}^{\top} \mathrm{d} W_{s}=: P_{t_{1}}^{t, p_{2}, \hat{\alpha}_{2}}
\end{array} .\right.
$$

In particular, thanks to Remark 4.5, we know that there exists $\tilde{\alpha}_{2} \in \mathcal{A}_{t, p_{2}}^{t_{2}}$ which coincides with $\hat{\alpha}_{2}$ on $\left[t, t_{1}\right]$ (see $[2$, Theorem 3.1]). In what follows we do not make the difference between $\tilde{\alpha}_{2}$ and $\hat{\alpha}_{2}$. 
1.c We denote $\widehat{\mathbf{P}}_{t_{1}}^{1}:=\left(P_{t_{1}}^{t, p_{1}, \hat{\alpha}_{1}}, P_{t_{1}}^{t, p_{2}, \hat{\alpha}_{2}}\right)$ and $\widehat{\mathbf{Q}}_{t_{1}}^{1}:=\left(\hat{q}_{1}\left(\hat{q}_{2}\right) Q_{t_{1}}^{t, x, 1}, \hat{q}_{2} Q_{t_{1}}^{t, x, 1}\right)$. The step 1.b and Remark 4.8 imply that $\hat{\mathbf{P}}_{t_{1}}^{1}$ lies in the sub-differential of $\left(w_{\mathrm{EU}}^{\sharp} \vee g\right)^{\sharp}\left(t_{1}, X_{t_{1}}^{t, x}, \cdot\right)$ at $\hat{\mathbf{Q}}_{t_{1}}^{1}$.

Hence using (4.12) with respectively $\psi=\left(w_{\mathrm{EU}}^{\sharp} \vee g\right)^{\sharp}$ and $\psi=w_{\mathrm{EU}}$ we can write

$$
\begin{aligned}
& \left(w_{\mathrm{EU}}^{\sharp} \vee g\right)^{\sharp \sharp}\left(t_{1}, X_{t_{1}}^{t, x}, P_{t_{1}}^{t, p_{1}, \hat{\alpha}_{1}}, P_{t_{1}}^{t, p_{2}, \hat{\alpha}_{2}}\right) \\
& \quad+\left(w_{\mathrm{EU}}^{\sharp} \vee g\right)^{\sharp}\left(t_{1}, X_{t_{1}}^{t, x}, \hat{q}_{1}\left(\hat{q}_{2}\right) Q_{t_{1}}^{t, x, 1}, \hat{q}_{2} Q_{t_{1}}^{t, x, 1}\right)=\widehat{\mathbf{P}}_{t_{1}}^{1} \widehat{\mathbf{Q}}_{t_{1}}^{1^{\top}},
\end{aligned}
$$

and

$$
w_{\mathrm{EU}}^{\sharp}\left(t, x, \hat{p}_{1}, \hat{p}_{2}\right)+w_{\mathrm{EU}}\left(t, x, \hat{q}_{1}, \hat{q}_{2}\right)=\hat{p}_{1} \hat{q}_{1}+\hat{p}_{2} \hat{q}_{2},
$$

where by (4.7),

$$
w_{\mathrm{EU}}\left(t, x, \hat{q}_{1}, \hat{q}_{2}\right)=\mathbb{E}^{\mathbb{Q}_{t, x}}\left[\left(w_{\mathrm{EU}}^{\sharp} \vee g\right)^{\sharp}\left(t_{1}, X_{t_{1}}^{t, x}, \hat{q}_{1}\left(\hat{q}_{2}\right) Q_{t_{1}}^{t, x, 1}, \hat{q}_{2} Q_{t_{1}}^{t, x, 1}\right)\right] .
$$

Thus inserting (4.20)-(4.21) and (4.24) into (4.23) and appealing to (4.22) we obtain

$$
\begin{aligned}
w_{\mathrm{EU}}^{\sharp}\left(t, x, \hat{p}_{1}, \hat{p}_{2}\right) & =\mathbb{E}^{\mathbb{Q} t, x}\left[\widehat{\mathbf{P}}_{t_{1}}^{1} \widehat{\mathbf{Q}}_{t_{1}}^{1^{\top}}-\left(w_{\mathrm{EU}}^{\sharp} \vee g\right)^{\sharp}\left(t_{1}, X_{t_{1}}^{t, x}, \hat{q}_{1}\left(\hat{q}_{2}\right) Q_{t_{1}}^{t, x, 1}, \hat{q}_{2} Q_{t_{1}}^{t, x, 1}\right)\right] \\
& =\mathbb{E}^{\mathbb{Q} t, x}\left[\left(w_{\mathrm{EU}}^{\sharp} \vee g\right)^{\sharp \sharp}\left(t_{1}, X_{t_{1}}^{t, x}, P_{t_{1}}^{t, p_{1}, \hat{\alpha}_{1}}, P_{t_{1}}^{t, p_{2}, \hat{\alpha}_{2}}\right)\right] .
\end{aligned}
$$

We conclude appealing to [20, Theorem 12.2].

2. We assume that $\left(p_{1}, p_{2}\right) \in\{1\} \times\{1\}$. It follows from Lemma 4.3 that $w_{\mathrm{EU}}^{\sharp}(t, x, 1,1)=$ $v_{\mathrm{BM}}(t, x, 1)$. Hence

$$
w_{\mathrm{EU}}^{\sharp}(t, x, 1,1)=\mathbb{E}^{\mathbb{Q}_{t, x}}\left[\left(v_{\mathrm{EU}} \vee g\right)\left(t_{1}, X_{t_{1}}^{t, x}, 1,1\right)\right]=\mathbb{E}^{\mathbb{Q}_{t, x}}\left[\overline{\operatorname{conv}}\left(w_{\mathrm{EU}}^{\sharp} \vee g\right)\left(t_{1}, X_{t_{1}}^{t, x}, 1,1\right)\right],
$$

thanks to (3.12) and Remark 4.5.

3. We now assume that $\left(p_{1}, p_{2}\right) \in[0,1) \times\{1\}$ or $\left(p_{1}, p_{2}\right) \in\{1\} \times[0,1)$.

We study the first case as the other one can be treated similarly. Repeating the arguments used in [2] or in the proof of Theorem 7.1 in the appendix, one can prove that, for $p=p_{1}$,

$$
\begin{aligned}
v_{\mathrm{EU}}(t, x, p, 1) & =\mathbb{E}^{\mathbb{Q}_{t, x}}\left[\overline{\operatorname{conv}}\left(v_{\mathrm{EU}} \vee g\right)\left(t_{1}, X_{t_{1}}^{t, x}, P_{t_{1}}^{t, p, \hat{\alpha}}, 1\right)\right] \\
& =\sup _{q \geqslant 0}\left\{p q-\mathbb{E}^{\mathbb{Q} t, x}\left[\left(v_{\mathrm{EU}} \vee g\right)^{\sharp}\left(t_{1}, X_{t_{1}}^{t, x}, q Q_{t_{1}}^{t, x, 1}, 1\right)\right]\right\},
\end{aligned}
$$

where $\hat{\alpha} \in \mathcal{A}_{t, p}^{t_{1}}$ comes from the Martingale Representation Theorem of $\left(\lambda \mathrm{D}_{q}^{+}\left(v_{\mathrm{EU}} \vee g\right)^{\sharp}+\beta(1-\lambda) \mathrm{D}_{q}^{-}\left(v_{\mathrm{EU}} \vee g\right)^{\sharp}\right)\left(t_{1}, X_{t_{1}}^{t, x}, \hat{q} Q_{t_{1}}^{t, x, 1}, 1\right)=p+\int_{t}^{t_{1}} \hat{\alpha}_{s}^{\top} \mathrm{d} W_{s}=: P_{t_{1}}^{t, p, \hat{\alpha}}$, 
where $\beta=\{0,1\}, \lambda \in[0,1]$ and $\hat{q} \geqslant 0$ are such that

$$
p=\mathbb{E}\left[\left(\lambda \mathrm{D}_{q}^{+}\left(v_{\mathrm{EU}} \vee g\right)^{\sharp}+\beta(1-\lambda) \mathrm{D}_{q}^{-}\left(v_{\mathrm{EU}} \vee g\right)^{\sharp}\right)\left(t_{1}, X_{t_{1}}^{t, x}, \hat{q} Q_{t_{1}}^{t, x, 1}, 1\right)\right] .
$$

On the other hand appealing to (4.7), Proposition 4.3 and Remark 4.5 one can prove that

$$
\begin{aligned}
w_{\mathrm{EU}}^{\sharp}\left(t, x, p_{1}, 1\right) & \geqslant \sup _{q_{1} \geqslant 0} \lim _{q_{2} \rightarrow \infty}\left\{p_{1} q_{1}+q_{2}-\mathbb{E}^{\mathbb{Q}_{t, x}}\left[\left(w_{\mathrm{EU}}^{\sharp} \vee g\right)^{\sharp}\left(t_{1}, X_{t_{1}}^{t, x}, q_{1} Q_{t_{1}}^{t, x, 1}, q_{2} Q_{t_{1}}^{t, x, 1}\right)\right]\right\} \\
& =\sup _{q_{1} \geqslant 0}\left\{p_{1} q_{1}-\mathbb{E}^{\mathbb{Q}_{t, x}}\left[\left(v_{\mathrm{EU}} \vee g\right)^{\sharp}\left(t_{1}, X_{t_{1}}^{t, x}, q_{1} Q_{t_{1}}^{t, x, 1}, 1\right)\right]\right\} .
\end{aligned}
$$

As a consequence it follows from (4.25) that $w_{\mathrm{EU}}^{\sharp}\left(t, x, p_{1}, 1\right) \geqslant v_{\mathrm{EU}}\left(t, x, p_{1}, 1\right)$. As we already have $w_{\mathrm{EU}}^{\sharp}\left(t, x, p_{1}, 1\right) \leqslant v_{\mathrm{EU}}\left(t, x, p_{1}, 1\right)$ (recall the discussion at the beginning of Section 4.3) this proves the required result.

As already mentioned, Remark 4.5, Proposition 4.4 and Theorem 4.2 imply the following proposition.

Proposition 4.5. On $\left[t_{0}, T\right] \times \mathcal{O}_{+}^{d} \times[0,1]^{2}, v_{\mathrm{EU}} \leqslant w_{\mathrm{EU}}^{\sharp}$.

\subsection{Numerical illustrations}

In this section, we present a numerical illustration for $v_{\mathrm{EU}}$ and provide some comparisons with $v_{\mathrm{BM}}$, defined in (3.13). We use the algorithm derived in (4.7) and in [2, eq. 2.22]. We now fix $T=1$ and $n=2$ and work in a Black-Scholes setting with market parameters: $d=1, \sigma(t, x)=0.25 x, \lambda(t, x)=0.2$. We consider the case where $g \equiv K$ with $K=10$ (i.e there is no dependency on $x$ ).

In figure 1 , we plot the functions $v_{\mathrm{EU}}$ and $v_{\mathrm{EU}}^{\sharp}$ at $t_{0}$. In figure 2 , we plot in (c) the function $v_{\mathrm{EU}}\left(t_{0}, \cdot, p_{2}\right)$ for different values of $p_{2}$, and we plot in (d) the function $v_{\mathrm{EU}}\left(t_{0}, p_{1}, \cdot\right)$ for different values of $p_{1}$. We notice the increasing property of the value function in $p_{1}$ and $p_{2}$. 


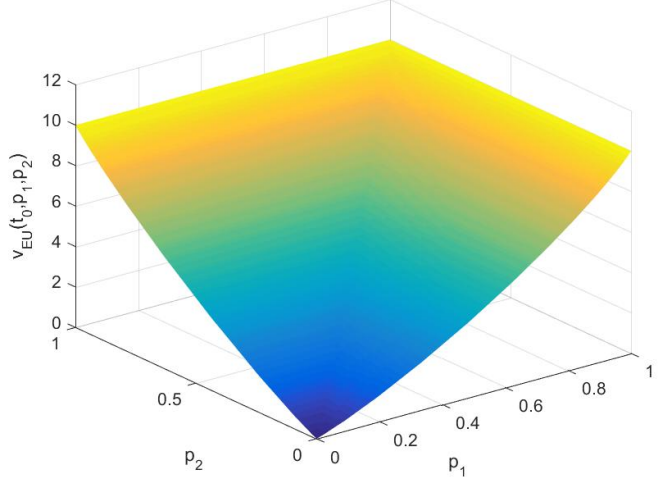

(a)

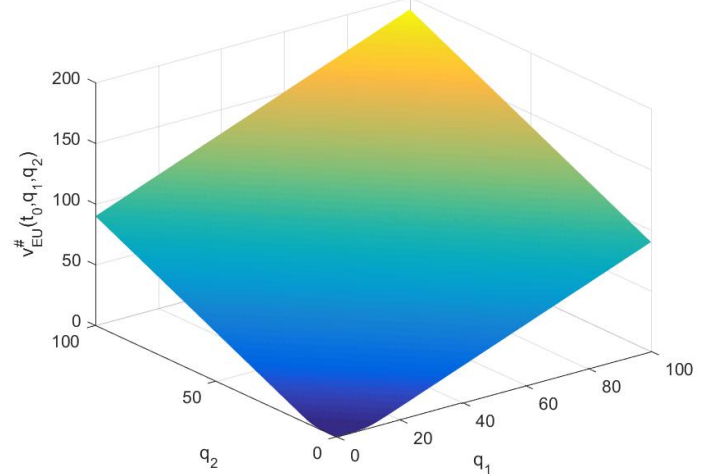

(b)

Figure 1: Surface of $v_{\mathrm{EU}}\left(t_{0}, p_{1}, p_{2}\right)$ and $v_{\mathrm{EU}}^{\sharp}\left(t_{0}, q_{1}, q_{2}\right)$.

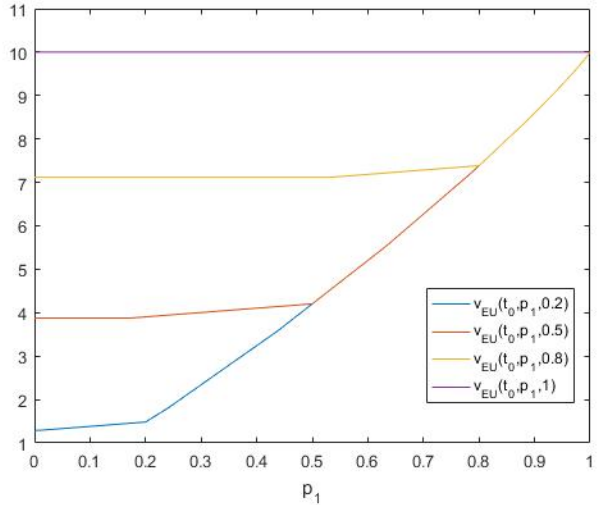

(c)

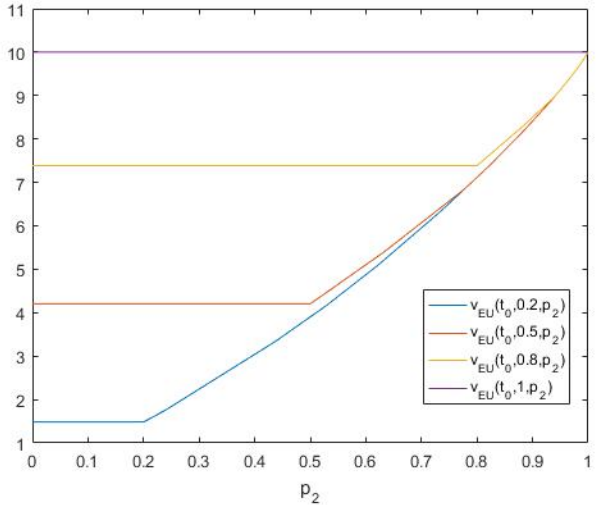

(d)

Figure 2: (c) Plot of $v_{\mathrm{EU}}\left(t_{0}, \cdot, p_{2}\right)$ for different values of $p_{2}$. (d) Plot of $v_{\mathrm{EU}}\left(t_{0}, p_{1}, \cdot\right)$ for different values of $p_{1}$.

Interestingly, one can prove appealing to Proposition 4.3 and [2, Proposition 3.3] 
and to the independence of $\lambda$ in $x$, that for $p \in[0,1]$ and $K>0$,

$$
\begin{aligned}
v_{\mathrm{EU}}\left(t_{0}, p, p\right) & =\sup _{\left(q_{1}, q_{2}\right) \in \mathbb{R}^{2}}\left\{\begin{array}{c}
p\left(q_{1}+q_{2}\right)-\mathbb{E}^{\mathbb{Q}_{t}}\left[\left[q_{2} Q_{t_{2}}^{t, 1}-K\right]^{+}\right] \mathbf{1}_{\left\{q_{1}=0\right\}} \\
-\mathbb{E}^{\mathbb{Q} t}\left[\left[\left(q_{1}+q_{2}\right) Q_{t_{1}}^{t, 1}-K\right] \mathbf{1}_{\left\{q_{1} Q_{t_{1}}^{t, 1} \geqslant K\right\}}\right] \\
-\mathbb{E}^{\mathbb{Q}_{t}}\left[\left[\left(q_{1}+q_{2}\right) Q_{t_{1}}^{t, 1}-K\right]^{+} \mathbf{1}_{\left\{0<q_{1} Q_{t_{1}}^{t, 1}<K\right\}}\right]
\end{array}\right\} \\
& \geqslant \sup _{q_{1} \geqslant 0}\left\{p q_{1}-\mathbb{E}^{\mathbb{Q}_{t}}\left[\left[q_{1} Q_{t_{1}}^{t, 1}-K\right]^{+}\right]\right\} \\
& =v_{\mathrm{BM}}\left(t_{0}, p\right),
\end{aligned}
$$

implying with Remark 5.1 (b) below that $v_{\mathrm{EU}}=v_{\mathrm{BM}}$.

\section{Problem 2: Hedging price under next-period quantile hedging constraints}

For the next-period constraint, we consider the quantile hedging constraint at each payment date given the market information at the previous time step. The dynamic programming principle is simplified as it only involves two successive dates (see Proposition 5.2). This structure will allow us to build the dual algorithm from the one holding in the case of a single European constraint (see Theorem 5.1). Though the analysis conducted in this section essentially follows from the previous section and [2], it provides a result that is still missing in the literature.

Let us define the problem we intend to solve.

On $\left[t_{i}, t_{i+1}\right) \times \mathcal{O}_{+}^{d} \times[0,1]^{n-i}, 0 \leqslant i \leqslant n-1$ we define

$$
v_{\mathrm{NP}}\left(t, x, p_{i+1}, \ldots, p_{n}\right):=\inf \Gamma_{\mathrm{NP}}\left(t, x, p_{i+1}, \ldots, p_{n}\right),
$$

with

$\Gamma_{\mathrm{NP}}\left(t, x, p_{i+1}, \ldots, p_{n}\right):=\left\{\begin{array}{c}y \geqslant 0: \exists \nu \in \mathcal{U}_{t, x, y} \text { s.t. } \mathbb{P}\left[\mathcal{S}_{t_{i+1}}^{t, x, y, \nu} \mid \mathcal{F}_{t}\right] \geqslant p_{i+1} \\ \text { and } \mathbb{P}\left[\mathcal{S}_{t_{k}}^{t, x, y, \nu} \mid \mathcal{F}_{t_{k-1}}\right] \geqslant p_{k}, i+2 \leqslant k \leqslant n, \text { if } i<n-1\end{array}\right\}$,

where we recall that $\mathcal{S}_{t_{k}}^{t, x, y, \nu}:=\left\{Y_{t_{k}}^{t, x, y, \nu} \geqslant g\left(t_{k}, X_{t_{k}}^{t, x}\right)\right\}, k \geqslant i+1$. On $\mathcal{O}_{+}^{d} \times[0,1]$, we set $v_{\mathrm{NP}}(T, \cdot)=0$.

Moreover, as in the previous section, we can also restrict to strategies such that $v_{\mathrm{NP}}$ satisfies a linear growth. Indeed (4.3) implies that we can find a constant $C>0$ on $\left[t_{i}, t_{i+1}\right) \times \mathcal{O}_{+}^{d} \times[0,1]^{n-i}, 0 \leqslant i \leqslant n-1$, such that

$$
0 \leqslant v_{\mathrm{NP}}\left(t, x, p_{i+1}, \ldots, p_{n}\right) \leqslant C(1+|x|) .
$$


Similarly to the previous section, we intend to build a backward dual representation of $v_{\mathrm{NP}}$.

We start with the following remark stating the relationship between the value function $v_{\mathrm{BM}}$ defined in (3.13), $v_{\mathrm{EU}}$ defined in (4.1) and $v_{\mathrm{NP}}$.

\section{Remark 5.1.}

(a) $O n\left[t_{n-1}, t_{n}\right) \times \mathcal{O}_{+}^{d}, v_{\mathrm{BM}}\left(t, x, p^{\prime}\right)=v_{\mathrm{EU}}\left(t, x, p^{\prime}\right)=v_{\mathrm{NP}}\left(t, x, p^{\prime}\right), p^{\prime} \in(-\infty, 1]$. Moreover on $\mathcal{O}_{+}^{d} \times[0,1], v_{\mathrm{BM}}(T, \cdot)=v_{\mathrm{EU}}(T, \cdot)=v_{\mathrm{NP}}(T, \cdot)=0$.

(b) $O n\left[t_{i}, t_{i+1}\right) \times \mathcal{O}_{+}^{d} \times(-\infty, 1], 0 \leqslant i \leqslant n-1, v_{\mathrm{BM}}\left(t, x, p^{\prime}\right) \geqslant v_{\mathrm{EU}}\left(t, x, p^{\prime}, \ldots, p^{\prime}\right)$ and $v_{\mathrm{NP}}\left(t, x, p^{\prime}, \ldots, p^{\prime}\right) \geqslant v_{\mathrm{EU}}\left(t, x, p^{\prime}, \ldots, p^{\prime}\right)$.

(c) $O n\left[t_{i}, t_{i+1}\right) \times \mathcal{O}_{+}^{d}, 0 \leqslant i \leqslant n-1, v_{\mathrm{BM}}(t, x, 1)=v_{\mathrm{EU}}(t, x, 1, \ldots, 1)=v_{\mathrm{NP}}(t, x, 1, \ldots, 1)$, $v_{\mathrm{BM}}(t, x, p)=v_{\mathrm{EU}}\left(t, x, p_{i+1}, \ldots, p_{n}\right)=v_{\mathrm{NP}}\left(t, x, p_{i+1}, \ldots, p_{n}\right)$ whenever $\left(p, p_{i+1}, \ldots, p_{n}\right) \in$ $(-\infty, 0]^{n-i+1}$ and $v_{\mathrm{BM}}(t, x, p)=v_{\mathrm{EU}}\left(t, x, p_{i+1}, \ldots, p_{n}\right)=v_{\mathrm{NP}}\left(t, x, p_{i+1}, \ldots, p_{n}\right)=\infty$ whenever $p>1$ and if there exists a $i+1 \leqslant k \leqslant n$ for which $p_{k}>1$.

We can now follow the arguments developed in the previous section and [2] to represent the value function $v_{\mathrm{NP}}$. More precisely, in Proposition 5.1, we will first reduce the problem to a stochastic target one of an American type (see [6]). This result will be useful to give a preliminary probabilistic representation of $v_{\mathrm{NP}}$ in Proposition 5.2. We will then be able to build in Theorem 5.1 the required algorithm allowing to compute $v_{\mathrm{NP}}$ by backward recursion.

\subsection{Equivalent formulation as a stochastic target problem}

We start with the problem reduction.

Proposition 5.1. On $\left[t_{i}, t_{i+1}\right) \times \mathcal{O}_{+}^{d} \times[0,1]^{n-i}, 0 \leqslant i \leqslant n-1$, we have

$\Gamma_{\mathrm{NP}}\left(t, x, p_{i+1}, \ldots, p_{n}\right)=\left\{\begin{array}{c}y \geqslant 0: \exists\left(\nu, \alpha_{i+1}\right) \in \mathcal{U}_{t, x, y} \times \mathcal{A}_{t, p_{i+1}}^{t_{i+1}} \text { s.t. } \\ Y_{t_{i+1}}^{t, x, y, \nu} \geqslant g\left(t_{i+1}, X_{t_{i+1}}^{t, x}, P_{t_{i+1}}^{t, p_{i+1}, \alpha_{i+1}}\right) \\ \text { and } \exists\left(\alpha_{i+2}, \ldots, \alpha_{n}\right) \in \mathcal{A}_{t_{i+1}, p_{i+2}}^{t_{i+2}} \times \ldots \times \mathcal{A}_{t_{n-1}, p_{n}}^{t_{n}} \text { s.t. } \\ Y_{t_{k}}^{t, x, y, \nu} \geqslant g\left(t_{k}, X_{t_{k}}^{t, x}, P_{t_{k}}^{t_{k-1}, p_{k}, \alpha_{k}}\right), i+2 \leqslant k \leqslant n, \text { if } i<n-1\end{array}\right\}$,

with the notations of (3.10) and (3.7).

Proof. The proof follows the same lines as those in [2, proof of Proposition 2.2], but we provide it for the sake of completeness. We fix $0 \leqslant i<n-1$ (as the case $i=n-1$ can be treated similarly) and consider $\left(t, x, p_{i+1}, \ldots, p_{n}\right) \in\left[t_{i}, t_{i+1}\right) \times \mathcal{O}_{+}^{d} \times[0,1]^{n-i}$. Let 
$\bar{\Gamma}\left(t, x, p_{i+1}, \ldots, p_{n}\right)$ denote the right-hand side in (5.3) and take $y$ as one of his elements. We can then find $\left(\nu, \alpha_{i+1}, \ldots, \alpha_{n}\right) \in \mathcal{U}_{t, x, y} \times \mathcal{A}_{t, p_{i+1}}^{t_{i+1}} \times \ldots \times \mathcal{A}_{t_{n-1}, p_{n}}^{t_{n}}$ such that

$Y_{t_{i+1}}^{t, x, y, \nu} \geqslant g\left(t_{i+1}, X_{t_{i+1}}^{t, x}, P_{t_{i+1}}^{t, p_{i+1}, \alpha_{i+1}}\right)$ and $Y_{t_{k}}^{t, x, y, \nu} \geqslant g\left(t_{k}, X_{t_{k}}^{t, x}, P_{t_{k}}^{t_{k-1}, p_{k}, \alpha_{k}}\right), i+2 \leqslant k \leqslant n$.

In particular for $i+2 \leqslant k \leqslant n$, the latter inequalities imply that $\mathcal{S}_{t_{k}}^{t, x, y, \nu} \supset\left\{P_{t_{k}}^{t_{k-1}, p_{k}, \alpha_{k}}>\right.$ $0\}$. Moreover, as $P^{t_{k-1}, p_{k}, \alpha_{k}} \in[0,1]$ on $\left[t_{k-1}, t_{k}\right]$, we have $\mathbf{1}_{\left\{P^{\left.t_{k-1}, p_{k}, \alpha_{k}>0\right\}}\right.} \geqslant P^{t_{k-1}, p_{k}, \alpha_{k}}$. Hence using the latter properties together with the martingale property of $P^{t_{k-1}, p_{k}, \alpha_{k}}$, we obtain

$$
\mathbb{P}\left[\mathcal{S}_{t_{k}}^{t, x, y, \nu} \mid \mathcal{F}_{t_{k-1}}\right] \geqslant \mathbb{P}\left[P_{t_{k}}^{t_{k-1}, p_{k}, \alpha_{k}}>0\right] \geqslant \mathbb{E}\left[P_{t_{k}}^{t_{k-1}, p_{k}, \alpha_{k}}\right]=p_{k} .
$$

Repeating the same arguments for $k=i+1$, we conclude that $y \in \Gamma_{\mathrm{NP}}\left(t, x, p_{i+1}, \ldots, p_{n}\right)$.

We now fix $y \in \Gamma_{\mathrm{NP}}\left(t, x, p_{i+1}, \ldots, p_{n}\right)$. We take $\nu \in \mathcal{U}_{t, x, y}$ such that

$$
p_{i+1}^{\prime}:=\mathbb{P}\left[\mathcal{S}_{t_{i+1}}^{t, x, y, \nu}\right] \geqslant p_{i+1} \text { and } p_{k}^{\prime}:=\mathbb{P}\left[\mathcal{S}_{t_{k}}^{t, x, y, \nu} \mid \mathcal{F}_{t_{k-1}}\right] \geqslant p_{k}, \forall i+2 \leqslant k \leqslant n .
$$

For $i+2 \leqslant k \leqslant n$, appealing to the Martingale Representation Theorem, we can find $\alpha_{k} \in \mathcal{A}$ such that

$$
\mathbf{1}_{\mathcal{S}_{t_{k}}^{t, x, y, \nu}}=: P_{t_{k}}^{t_{k-1}, p_{k}^{\prime}, \alpha_{k}} \geqslant P_{t_{k}}^{t_{k-1}, p_{k}, \alpha_{k}} .
$$

By replacing $\alpha_{k}$ by the constant null process after the first time after $t_{k-1}$ at which $P^{t_{k-1}, p_{k}, \alpha_{k}}$ reaches the level 0 , we can consider that $\alpha_{k} \in \mathcal{A}_{t_{k-1}, p_{k}}^{t_{k}}$. We can also notice that (5.4) is equivalent to $Y_{t_{k}}^{t, x, y, \nu} \geqslant g\left(t_{k}, X_{t_{k}}^{t, x}, P_{t_{k}}^{t_{k-1}, p_{k}, \alpha_{k}}\right)$. Repeating the same arguments for $k=i+1$, we conclude that $y \in \bar{\Gamma}\left(t, x, p_{i+1}, \ldots, p_{n}\right)$.

\subsection{Dynamic programming and backward dual algorithm}

As noticed earlier, the formulation obtained in Proposition 5.1 allows to derive a first dynamic programming algorithm. We omit the proof as it is standard (see [2, proof of Proposition 2.3]). It appeals, in particular, to (3.8)-(3.9) and (5.2).

Proposition 5.2. Fix $\left(t, x, p_{i+1}, \ldots, p_{n}\right) \in\left[t_{i}, t_{i+1}\right) \times \mathcal{O}_{+}^{d} \times[0,1]^{n-i}, 0 \leqslant i \leqslant n-1$,

$$
\begin{aligned}
& v_{\mathrm{NP}}\left(t, x, p_{i+1}, \ldots, p_{n}\right) \\
& =\left\{\begin{array}{ll}
\inf _{\alpha \in \mathcal{A}_{t, p_{i+1}}^{t_{i+1}}} \mathbb{E}^{\mathbb{Q} t, x}\left[\left(v_{\mathrm{NP}} \vee g\right)\left(t_{i+1}, X_{t_{i+1}}^{t, x}, P_{t_{i+1}}^{t, p_{i+1}, \alpha}, p_{i+2}, \ldots, p_{n}\right)\right], \\
\inf _{\alpha \in \mathcal{A}_{t, p_{n}}^{t_{n}}} \mathbb{E}^{\mathbb{Q}_{t, x}}\left[g\left(t_{n}, X_{t_{n}}^{t, x}, P_{t_{n}}^{t, p_{n}, \alpha}\right)\right], & \text { if } i<n-n-1
\end{array},\right.
\end{aligned}
$$


where we used the notations given by (3.11) with $f \equiv v_{\mathrm{NP}}$ and (3.7).

As a consequence there exists a constant $C>0$ such that for all $\left(t, x, x^{\prime}, p_{i+1}, \ldots, p_{n}\right) \in$ $\left[t_{i}, t_{i+1}\right) \times\left(\mathcal{O}_{+}^{d}\right)^{2} \times[0,1]^{n-i}, 0 \leqslant i \leqslant n-1$,

$$
\left|v_{\mathrm{NP}}\left(t, x, p_{i+1}, \ldots, p_{n}\right)-v_{\mathrm{NP}}\left(t, x^{\prime}, p_{i+1}, \ldots, p_{n}\right)\right| \leqslant C\left(1+|x|+\left|x^{\prime}\right|\right)\left|x-x^{\prime}\right| .
$$

We can now state the main theorem of this part which will allow to compute $v_{\mathrm{NP}}$ defined in Proposition 5.2. It involves the following algorithm defined on $\mathcal{O}_{+}^{d} \times \mathbb{R}$ and for $\left(p_{i+2}, \ldots, p_{n}\right) \in(-\infty, 1]^{n-i-1}, i<n-1$ by,

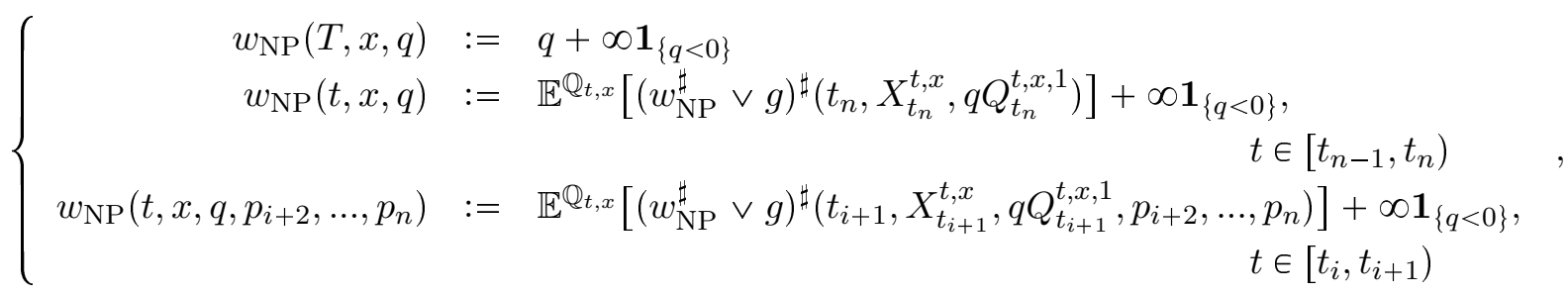

where $\left(w_{\mathrm{NP}}^{\sharp} \vee g\right)^{\sharp}$ is the Legendre-Fenchel transform of $\left(w_{\mathrm{NP}}^{\sharp} \vee g\right)$ with respect to the third variable. In particular, one can easily compute

$$
\begin{aligned}
& \mathbb{E}^{\mathbb{Q} t, x}\left[\left(w_{\mathrm{NP}}^{\sharp} \vee g\right)^{\sharp}\left(t_{i+1}, X_{i+1}^{t, x}, q Q_{t_{i+1}}^{t, x, 1}, p_{i+2}, \ldots, p_{n}\right)\right] \\
&= {\left[q Q_{t_{i+1}}^{t, x, 1}-\left[g\left(t_{i+1}, X_{t_{i+1}}^{t, x}\right)-w_{\mathrm{NP}}^{\sharp}\left(t_{i+1}, X_{t_{i+1}}^{t, x}, p_{i+2}, \ldots, p_{n}\right)\right]^{+}\right]^{+} } \\
&-w_{\mathrm{NP}}^{\sharp}\left(t_{i+1}, X_{t_{i+1}}^{t, x}, p_{i+2}, \ldots, p_{n}\right),
\end{aligned}
$$

where, for all $x \in \mathcal{O}_{+}^{d}$ and $\left(p_{i+2}, \ldots, p_{n}\right) \in(-\infty, 1]^{n-i-1}, i<n-1$,

$w_{\mathrm{NP}}^{\sharp}\left(t_{i+1}, x, p_{i+2}, \ldots, p_{n}\right):=\left\{\begin{array}{l}\sup _{q \in \mathbb{R}}\left\{p_{i+2} q-w_{\mathrm{NP}}\left(t_{i+1}, x, q, p_{i+3}, \ldots, p_{n}\right)\right\}, \text { if } i<n-2 \\ \sup _{q \in \mathbb{R}}\left\{p_{i+2} q-w_{\mathrm{NP}}\left(t_{i+1}, x, q\right)\right\}, \text { if } i=n-2\end{array}\right.$

Theorem 5.1. Fix $0 \leqslant i \leqslant n-1$. On $\left[t_{i}, t_{i+1}\right) \times \mathcal{O}_{+}^{d} \times[0,1]^{n-i}$, we have $v_{\mathrm{NP}}=w_{\mathrm{NP}}^{\sharp}$ (recall (5.5)). In particular,

$$
\begin{aligned}
& v_{\mathrm{NP}}\left(t, x, p_{i+1}, \ldots, p_{n}\right) \\
& \quad=\left\{\begin{array}{l}
\mathbb{E}^{\mathbb{Q}_{t, x}}\left[\overline{\operatorname{conv}}\left(v_{\mathrm{NP}} \vee g\right)\left(t_{i+1}, X_{t_{i+1}}^{t, x}, P_{t_{i+1}}^{t, p_{i+1}, \hat{\alpha}}, p_{i+2}, \ldots, p_{n}\right)\right], \text { if } i<n-1 \\
\mathbb{E}^{\mathbb{Q}_{t, x}}\left[P_{t_{n}}^{t, p_{n}, \hat{\alpha}} g\left(t_{n}, X_{t_{n}}^{t, x}\right)\right], \text { if } i=n-1
\end{array}\right.
\end{aligned}
$$

where $\overline{\operatorname{conv}}\left(v_{\mathrm{NP}} \vee g\right)$ is the closed convex envelope of ( $\left.v_{\mathrm{NP}} \vee g\right)$ ) (with respect to the third variable) given by (3.12) with $f \equiv v_{\mathrm{NP}}$, and where (omitting the dependencies of 
the variables involved) for $p_{i+1}=1, \hat{\alpha}=0$ while for $p_{i+1} \in[0,1), \hat{\alpha}$ comes from the martingale representation of

$$
\begin{aligned}
P_{t_{i+1}}^{t, p_{i+1}, \hat{\alpha}}:= & \lambda \mathbf{1}_{\left\{\hat{q} Q_{t_{i+1}}^{t, x, 1} \geqslant\left[g\left(t_{i+1}, X_{t_{i+1}}^{t, x}\right)-v_{\mathrm{NP}}\left(t_{i+1}, X_{t_{i+1}}^{t, x}, p_{i+2}, \ldots, p_{n}\right)\right]^{+}\right\}} \\
& +\beta(1-\lambda) \mathbf{1}_{\left\{\hat{q} Q_{t_{i+1}}^{t, x, 1}>\left[g\left(t_{i+1}, X_{t_{i+1}}^{t, x}\right)-v_{\mathrm{NP}}\left(t_{i+1}, X_{t_{i+1}}^{t, x}, p_{i+2}, \ldots, p_{n}\right)\right]^{+}\right\}}, \text {if } i<n-1
\end{aligned}
$$

(recall (5.6)), and of

$$
P_{t_{i+1}}^{t, p_{i+1}, \hat{\alpha}}:=\lambda \mathbf{1}_{\left\{\hat{q} Q_{t_{i+1}}^{t, x, 1} \geqslant g\left(t_{i+1}, X_{t_{i+1}}^{t, x}\right)\right\}}+\beta(1-\lambda) \mathbf{1}_{\left\{\hat{q} Q_{t_{i+1}}^{t, x, 1}>g\left(t_{i+1}, X_{t_{i+1}}^{t, x}\right)\right\}}, \text { if } i=n-1,
$$

and with $\hat{q} \geqslant 0, \lambda \in[0,1], \beta \in\{0,1\}$ being such that $\mathbb{E}\left[P_{t_{i+1}}^{t, p_{i+1}, \hat{\alpha}}\right]=p_{i+1}$.

Proof. The theorem is proved recursively backward using Proposition 5.2, (3.8)-(3.9) and (5.2) and adapting Theorem 7.1 in the appendix.

\subsection{Numerical illustrations}

In this section, we present a numerical illustration for $v_{\mathrm{NP}}$ and provide some comparisons with $v_{\mathrm{BM}}$, defined in (3.13). We use the algorithm derived in (5.5) and in [2, eq. 2.22]. We work under the same framework as the one in Section 4.4 and consider a put option, i.e. $g(t, x)=[K-x]^{+}$, with strike $K=30$.

In figure 3 , we plot the functions $v_{\mathrm{NP}}$ and $v_{\mathrm{NP}}^{\sharp}$ at $t_{0}$ and for $p_{2}=0.3$. In figure 4 , we plot in (c) the function $v_{\mathrm{NP}}\left(t_{0}, \cdot, p_{1}, p_{2}\right)$ for different values of $p_{1}$ and $p_{2}$, and we plot in (d) the functions $v_{\mathrm{NP}}\left(t_{0}, \cdot, p, p\right)$ and $v_{\mathrm{BM}}\left(t_{0}, \cdot, p\right)$ when $p=0.8$ and $p=1$. Figure 4 shows the decrease of value for both value functions, when $p$ decreases.

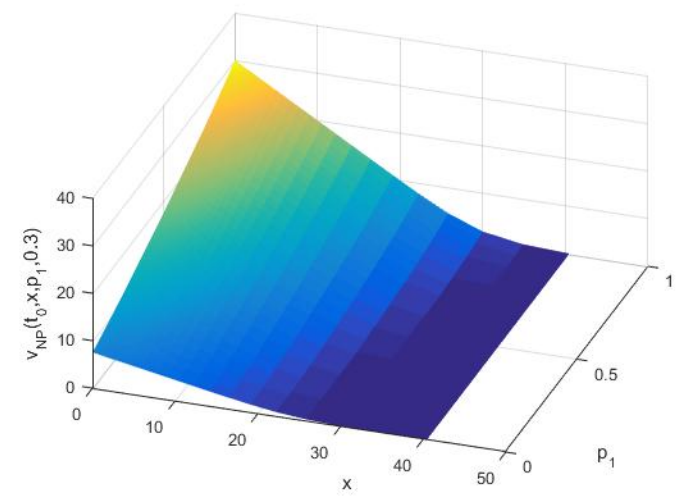

(a)

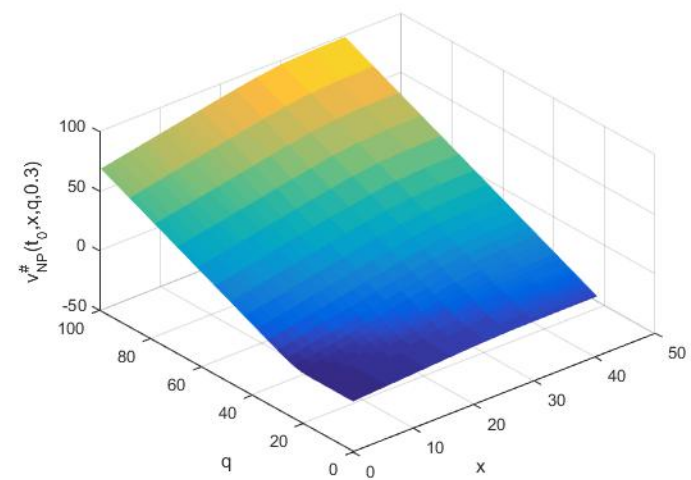

(b)

Figure 3: Surface of $v_{\mathrm{NP}}\left(t_{0}, x, p_{1}, 0.3\right)$ and $v_{\mathrm{NP}}^{\sharp}\left(t_{0}, x, q, 0.3\right)$. 


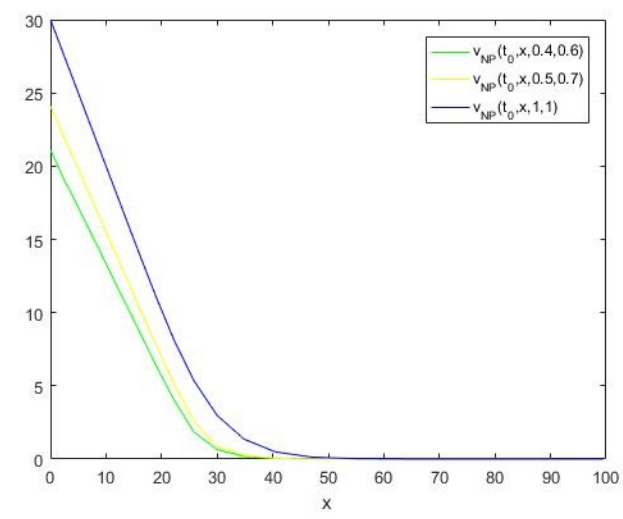

(c)

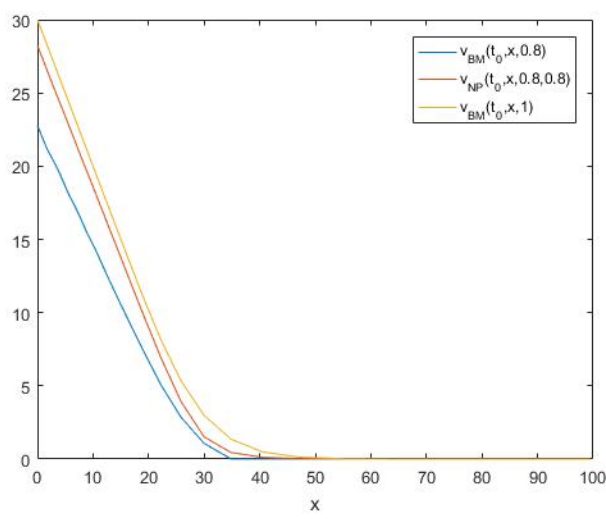

(d)

Figure 4: (c) Plot of $v_{\mathrm{NP}}\left(t_{0}, \cdot, p_{1}, p_{2}\right)$ for different values of $p_{1}$ and $p_{2}$. (d) Plot of $v_{\mathrm{NP}}\left(t_{0}, \cdot, p, p\right)$ and $v_{\mathrm{BM}}\left(t_{0}, \cdot, p\right)$ for $p=1$ and $p=0.8$.

\section{Problem 3: Cost of designing a portfolio satisfying an American expected utility-based constraint}

We want to characterize the cost of designing a portfolio that must satisfy at all random payment dates an expected utility-based constraint set at inception and involving a constant benchmark above which the portfolio process must always stay. The difficulty relies on handling the randomness of the dates at which the constraint holds. The loss function satisfies some regularity assumptions as it will be made clear in the next paragraph. Let us describe the problem we are interested in.

We fix $K \in \mathbb{R}$. In what follows, the dependency in $K$ will not always be made explicit to alleviate notations. We work under the following assumption.

Assumption 6.0.1. We consider a function $\Psi$ such that on $[K, \infty)$,

(a) $\operatorname{Im}(\Psi)=[\Psi(K), \infty) \subseteq \mathbb{R}^{+}$;

(b) $\Psi$ is continuous and increasing;

(c) for $(t, x, y) \in[0, T] \times \mathcal{O}_{+}^{d} \times[K, \infty)$ and $\nu \in \tilde{\mathcal{U}}_{t, x, y}^{K}$ (with $\tilde{\mathcal{U}}_{t, x, y}^{K}$ defined in Section $3), \mathbb{E}\left[\left|\Psi\left(Y^{t, x, y, \nu}\right)\right|^{2}\right]<\infty$ on $[t, T]$ and $\Psi\left(Y_{s}^{t, x, y, \nu}\right)$ is upper bounded uniformly in $s \in[t, T]$ by an integrable variable;

(d) its inverse $\Phi: p \in \operatorname{Im}(\Psi) \mapsto \Psi^{-1}(p) \in[K, \infty)$ is differentiable of differentiate continuous and strictly monotone and of inverse $\mathrm{I}: q \in[0, \infty) \mapsto\left(\mathrm{D}_{p} \Phi(\cdot)\right)^{-1}(q) \in$ $\operatorname{Im}(\Psi)$ 
(e) for $(t, x, q) \in[0, T] \times \mathcal{O}_{+}^{d} \times[0, \infty), \mathbb{E}\left[\left|\mathrm{I}\left(q Q^{t, x, 1}\right)\right|^{2}\right]<\infty$ on $[t, T]$ and $\mathrm{I}\left(q Q_{s}^{t, x, 1}\right)$ is upper bounded uniformly in $s \in[t, T]$ by an integrable variable;

(f) for $(t, x, q) \in[0, T] \times \mathcal{O}_{+}^{d} \times[0, \infty), \mathbb{E}^{\mathbb{Q}_{t, x}}\left[\left|\Phi\left(\mathrm{I}\left(q Q^{t, x, 1}\right)\right)\right|^{2}\right]<\infty$ on $[t, T]$.

By convention we set $\Phi(p)=+\infty$ for $p \notin \operatorname{Im}(\Psi)$.

In addition, we introduce for $(t, p) \in[0, T] \times \operatorname{Im}(\Psi)$ the set

$$
\tilde{\mathcal{A}}_{t, p}:=\left\{\alpha \in \mathcal{A} \text { s.t. } P^{t, p, \alpha}:=p+\int_{t}^{\cdot} \alpha_{v}^{\top} \mathrm{d} W_{v} \in \operatorname{Im}(\Psi) \text { on }[t, T]\right\} .
$$

Let us define on $[0, T] \times \mathcal{O}_{+}^{d} \times \operatorname{Im}(\Psi)$,

$$
v_{\mathrm{US}}(t, x, p):=\inf \Gamma_{\mathrm{US}}(t, x, p),
$$

with

$$
\Gamma_{\mathrm{US}}(t, x, p):=\left\{y \geqslant K: \exists \nu \in \tilde{\mathcal{U}}_{t, x, y}^{K} \text { s.t. } \mathbb{E}\left[\Psi\left(Y_{\tau}^{t, x, y, \nu}\right)\right] \geqslant p, \forall \tau \in \mathcal{T}_{[t, T]}\right\} .
$$

Remark 6.1. As mentioned in the introduction, this problem extends the analysis in [13] to the case where a utility-based risk measure constraint also holds.

We can now follow the same route as in [2] to represent the value function $v_{\text {US }}$. Namely, in Proposition 6.1, we will express the problem as a stochastic target problem of an American type (see [6]). This result will be important to prove a probabilistic representation of $v_{\text {US }}$ in Theorem 6.1. In particular, we will work towards the identification of the optimal control. It will involve the Legendre-Fenchel transform of $\Phi$.

\subsection{Equivalent formulation as a stochastic target problem}

We start with the problem reduction.

Proposition 6.1. Fix $(t, x, p) \in[0, T] \times \mathcal{O}_{+}^{d} \times \operatorname{Im}(\Psi)$, then

$$
\Gamma_{\mathrm{US}}(t, x, p)=\left\{y \geqslant K: \exists(\nu, \alpha) \in \tilde{\mathcal{U}}_{t, x, y}^{K} \times \tilde{\mathcal{A}}_{t, p} \text { s.t. } Y_{\tau}^{t, x, y, \nu} \geqslant \Phi\left(P_{\tau}^{t, p, \alpha}\right), \forall \tau \in \mathcal{T}_{[t, T]}\right\}
$$

(recall (6.1)). 
Proof. Fix $(t, x, p) \in[0, T] \times \mathcal{O}_{+}^{d} \times \operatorname{Im}(\Psi)$. Let $\bar{\Gamma}(t, x, p)$ denote the right-hand side of (6.2) and let $y$ be one of his elements. Fix $(\nu, \alpha) \in \tilde{\mathcal{U}}_{t, x, y}^{K} \times \tilde{\mathcal{A}}_{t, p}$. Then

$$
Y_{\tau}^{t, x, y, \nu} \geqslant \Phi\left(P_{\tau}^{t, p, \alpha}\right), \forall \tau \in \mathcal{T}_{[t, T]}
$$

Using the increasing property of $\Psi$ we obtain

$$
\Psi\left(Y_{\tau}^{t, x, y, \nu}\right) \geqslant P_{\tau}^{t, p, \alpha}, \forall \tau \in \mathcal{T}_{[t, T]} .
$$

We finally take the expectation and use the martingale property of $P^{t, p, \alpha}$ to obtain the result.

We now fix $y \in \Gamma_{\mathrm{US}}(t, x, p)$ and $\nu \in \tilde{\mathcal{U}}_{t, x, y}^{K}$ such that

$$
\mathbb{E}\left[\Psi\left(Y_{\tau}^{t, x, y, \nu}\right)\right] \geqslant p, \forall \tau \in \mathcal{T}_{[t, T]} .
$$

The inequality (6.3) is equivalent to

$$
\inf _{\tau \in \mathcal{T}_{[t, T]}} \mathbb{E}\left[\Psi\left(Y_{\tau}^{t, x, y, \nu}\right)\right] \geqslant p
$$

We set $\tilde{S}^{t, x, y, \nu}:=\Psi\left(Y^{t, x, y, \nu}\right)$ and define $Z^{t, x, y, \nu}:=\operatorname{essinf}_{\theta \in \mathcal{T}_{[\cdot, T]}} \mathbb{E}\left[\tilde{S}_{\theta}^{t, x, y, \nu} \mid \mathcal{F}\right.$. $]$ on all the stopping times in $\mathcal{T}_{[t, T]}$, with $\mathrm{Z}_{t}^{t, x, y, \nu}:=p^{\prime} \geqslant p$. Thanks to Assumption 6.0.1, (a)-(c) there exists a right-continuous and non-negative sub-martingale $\bar{Z}^{t, x, y, \nu}$ which aggregates $\mathrm{Z}^{t, x, y, \nu}$ in the sense that $\overline{\mathrm{Z}}_{\tau}^{t, x, y, \nu}=\mathrm{Z}_{\tau}^{t, x, y, \nu}$ for all $\tau \in \mathcal{T}_{[t, T]}$ (see e.g. [12, Section 2.15]). We will not make the difference between $\bar{Z}^{t, x, y, \nu}$ and $Z^{t, x, y, \nu}$. It follows from the Doob-Meyer decomposition (see Karatzas and Shreve [17, Chapter 1, Theorem 4.10]) that there exists a right-continuous martingale $M^{t, x, y, \nu}:=\left\{M_{s}^{t, x, y, \nu}, \mathcal{F}_{s}, t \leqslant s \leqslant\right.$ $T\}$ issued from $p^{\prime}$ at $t$ and an increasing natural process $A^{t, x, y, \nu}:=\left\{A_{s}^{t, x, y, \nu}, \mathcal{F}_{s}, t \leqslant\right.$ $s \leqslant T\}$ starting from 0 at $t$ such that $\mathrm{Z}_{\tau}^{t, x, y, \nu}=M_{\tau}^{t, x, y, \nu}+A_{\tau}^{t, x, y, \nu}, \forall \tau \in \mathcal{T}_{[t, T]}$. Hence $\forall \tau \in \mathcal{T}_{[t, T]}$,

$$
P_{\tau}^{t, p, \hat{\alpha}} \leqslant P_{\tau}^{t, p^{\prime}, \hat{\alpha}}:=M_{\tau}^{t, x, y, \nu} \leqslant \mathrm{Z}_{\tau}^{t, x, y, \nu} \leqslant \Psi\left(Y_{\tau}^{t, x, y, \nu}\right)
$$

with $\hat{\alpha}$ a square integrable process valued in $\mathbb{R}^{d}$ and such that $P^{t, p, \hat{\alpha}} \in \mathbb{R}$. By possibly replacing $\hat{\alpha}$ by the constant process 0 after the first time after $t$ at which $P^{t, p, \hat{\alpha}}$ reaches $\Psi(K)$, we can assume that $\hat{\alpha} \in \tilde{\mathcal{A}}_{t, p}$, and obtain after appealing to the non-decreasing property of $\Phi$ that $\forall \tau \in \mathcal{T}_{[t, T]}$,

$$
\Phi\left(P_{\tau}^{t, p, \hat{\alpha}}\right) \leqslant Y_{\tau}^{t, x, y, \nu}
$$

As a result $y \in \bar{\Gamma}(t, x, p)$. 


\subsection{A closed-form representation of $v_{\mathrm{US}}$}

As mentioned earlier, with the definition obtained in Proposition 6.1 at hand, we can now prove a probabilistic representation of the solution and characterize the optimum reached.

To this aim we focus on the dual $\Phi^{\sharp}$ of $\Phi$. By definition for $q \in[0, \infty)$ we have $\Phi^{\sharp}(q):=\sup _{p \in \mathbb{R}}\{p q-\Phi(p)\}=\sup _{p \in \operatorname{Im}(\Psi)}\{p q-\Phi(p)\}$, as by convention $\Phi(p)=+\infty$ for $p \notin \operatorname{Im}(\Psi)$. We deduce from the previous definition that for all $q \in[0, \infty)$,

$$
\left\{\begin{array}{l}
\Phi^{\sharp}(q) \geqslant p q-\Phi(p) \forall p \in \operatorname{Im}(\Psi) \\
\Phi^{\sharp}(q)=\mathrm{I}(q) q-\Phi(\mathrm{I}(q))
\end{array} .\right.
$$

Let us prove the main result of this part.

Theorem 6.1. Fix $(t, x, p) \in[0, T] \times \mathcal{O}_{+}^{d} \times \operatorname{Im}(\Psi)$. Then,

$$
v_{\mathrm{US}}(t, x, p)=\sup _{\tau \in \mathcal{T}_{[t, T]}} \mathbb{E}^{\mathbb{Q}_{t, x}}\left[\Phi\left(\operatorname{essinf}_{\theta \in \mathcal{T}_{[\tau, T]}} \mathbb{E}\left[\mathrm{I}\left(\tilde{q}^{\theta} Q_{\theta}^{t, x, 1}\right) \mid \mathcal{F}_{\tau}\right]\right)\right],
$$

with, for each $\tau, \tilde{q}^{\tau}:=\tilde{q}^{\tau}(t, x, p) \in[0, \infty)$ being deterministic and such that

$$
p=\mathbb{E}\left[\mathrm{I}\left(\tilde{q}^{\tau} Q_{\tau}^{t, x, 1}\right)\right] .
$$

In particular there exists $\hat{\alpha} \in \tilde{\mathcal{A}}_{t, p}$ (recall (6.1)) such that

$$
v_{\mathrm{US}}(t, x, p)=\sup _{\tau \in \mathcal{T}_{[t, T]}} \mathbb{E}^{\mathbb{Q}_{t, x}}\left[\Phi\left(P_{\tau}^{t, p, \hat{\alpha}}\right)\right] .
$$

Proof. Fix $(t, x, p) \in[0, T] \times \mathcal{O}_{+}^{d} \times \operatorname{Im}(\Psi)$.

1. Proof of (6.5).

We denote by $v_{1}$ the right-hand side of (6.5).

1.a Proof of $v_{\mathrm{US}} \leqslant v_{1}$.

Let $y>v_{1}$. We define $Z^{t, x}:=\operatorname{essinf}_{\theta \in \mathcal{T}_{[\cdot, T]}} \mathbb{E}\left[\mathrm{I}\left(\tilde{q}^{\theta} Q_{\theta}^{t, x, 1}\right) \mid \mathcal{F}\right.$. $]$ on all the stopping times in $\mathcal{T}_{[t, T]}$, with $\mathrm{Z}_{t}^{t, x}=p$. Thanks to Assumption 6.0.1, (a), (d) and (e), there exists a right-continuous and non-negative sub-martingale $\bar{Z}^{t, x}$ which aggregates $\mathrm{Z}^{t, x}$ in the sense that $\overline{\mathrm{Z}}_{\tau}^{t, x}=\mathrm{Z}_{\tau}^{t, x}$ for all $\tau \in \mathcal{T}_{[t, T]}$ (see e.g. [12, Section 2.15]). We will not make the difference between $\bar{Z}^{t, x}$ and $\mathrm{Z}^{t, x}$. It follows from the Doob-Meyer decomposition (see [17, Chapter 1, Theorem 4.10]) that there exists a right-continuous martingale $M^{t, x}:=\left\{M_{s}^{t, x}, \mathcal{F}_{s}, t \leqslant s \leqslant T\right\}$ issued from $p$ at $t$ and an increasing natural process $A^{t, x}:=\left\{A_{s}^{t, x}, \mathcal{F}_{s}, t \leqslant s \leqslant T\right\}$ starting from 0 at $t$ such that $\mathrm{Z}_{\tau}^{t, x}=M_{\tau}^{t, x}+A_{\tau}^{t, x}, \forall \tau \in$ $\mathcal{T}_{[t, T]}$. Hence $\forall \tau \in \mathcal{T}_{[t, T]}$,

$$
P_{\tau}^{t, p, \hat{\alpha}}:=M_{\tau}^{t, x} \leqslant \mathrm{Z}_{\tau}^{t, x} \leqslant \mathrm{I}\left(\tilde{q}^{\tau} Q_{\tau}^{t, x, 1}\right),
$$


with $\hat{\alpha}$ a square integrable process valued in $\mathbb{R}^{d}$ and such that $P^{t, p, \hat{\alpha}} \in \mathbb{R}$. By possibly replacing $\hat{\alpha}$ by the constant process 0 after the first time after $t$ at which $P^{t, p, \hat{\alpha}}$ reaches $\Psi(K)$, we can assume that $\hat{\alpha} \in \tilde{\mathcal{A}}_{t, p}$ and then use the non-decreasing property of $\Phi$ to obtain

$$
y \geqslant \mathbb{E}^{\mathbb{Q}_{t, x}}\left[\Phi\left(P_{\tau}^{t, p, \hat{\alpha}}\right)\right], \forall \tau \in \mathcal{T}_{[t, T]} .
$$

Therefore, appealing to Assumption 6.0.1, (f), and the definition of $\hat{\alpha} \in \tilde{\mathcal{A}}_{t, p}$, there exists $\hat{\nu} \in \tilde{\mathcal{U}}_{t, x, y}^{K}$ such that $\forall \tau \in \mathcal{T}_{[t, T]}$,

$$
Y_{\tau}^{t, x, y, \hat{\nu}} \geqslant \Phi\left(P_{\tau}^{t, p, \hat{\alpha}}\right) \geqslant K
$$

which, by Proposition 6.1, gives $y>v_{\mathrm{US}}$.

1.b Proof of $v_{\mathrm{US}} \geqslant v_{1}$.

Let $y>v_{\mathrm{US}}(t, x, p)$. It follows from Proposition 6.1 that there exists $(\nu, \alpha) \in \tilde{\mathcal{U}}_{t, x, y}^{K} \times \tilde{\mathcal{A}}_{t, p}$ such that for all $\tau \in \mathcal{T}_{[t, T]}$,

$$
Y_{\tau}^{t, x, y, \nu} \geqslant \Phi\left(P_{\tau}^{t, p, \alpha}\right)
$$

Using (6.4) and the non-decreasing property of $\Phi$ we obtain for all $\tau \in \mathcal{T}_{[t, T]}$,

$$
\begin{aligned}
Y_{\tau}^{t, x, y, \nu} & \geqslant \tilde{q}^{\tau} Q_{\tau}^{t, x, 1} P_{\tau}^{t, p, \alpha}-\tilde{q}^{\tau} Q_{\tau}^{t, x, 1} \mathrm{I}\left(\tilde{q}^{\tau} Q_{\tau}^{t, x, 1}\right)+\Phi\left(\mathrm{I}\left(\tilde{q}^{\tau} Q_{\tau}^{t, x, 1}\right)\right) \\
& \geqslant \tilde{q}^{\tau} Q_{\tau}^{t, x, 1} P_{\tau}^{t, p, \alpha}-\tilde{q}^{\tau} Q_{\tau}^{t, x, 1} \mathrm{I}\left(\tilde{q}^{\tau} Q_{\tau}^{t, x, 1}\right)+\Phi\left(\mathrm{Z}_{\tau}^{t, x}\right)
\end{aligned}
$$

with $\tilde{q}^{\tau}$ defined in (6.6) and $\mathrm{Z}^{t, x}$ defined in the step 1.a. Using (3.4), the $\mathbb{Q}_{t, x}$-supermartingale property of $Y^{t, x, y, \nu}$, the definition of $\tilde{q}^{\tau}(t, x, p)$ and of $\tilde{\mathcal{A}}_{t, p}$, we obtain taking the expectation under $\mathbb{Q}_{t, x}$,

$$
y \geqslant \mathbb{E}^{\mathbb{Q} t, x}\left[\Phi\left(\mathrm{Z}_{\tau}^{t, x}\right)\right], \forall \tau \in \mathcal{T}_{[t, T]},
$$

leading to the required result.

2. Proof of (6.7).

We denote by $v_{2}$ the right-hand side of (6.7).

Arguing as in the step 1.a up to (6.8) and using the relation $v_{\mathrm{US}}(t, x, p)=v_{1}(t, x, p)$, we obtain that $v_{\mathrm{US}}(t, x, p) \geqslant v_{2}(t, x, p)$. The other inequality is proved by appealing to the Martingale Representation Theorem to represent I $\left(\tilde{q}^{\tau} Q_{\tau}^{t, x, 1}\right)$ in (6.6) and the non-decreasing property of $\Phi$.

\section{Appendix}

We provide the proof of a technical result that is used in Section 5. 
Theorem 7.1. We consider the set-up defined in Section 3. We define on $\left[t_{0}, t_{1}\right) \times$ $\mathcal{O}_{+}^{d} \times[0,1]$ and for a given function $h:(t, x) \in[0, T] \times \mathcal{O}_{+}^{d} \mapsto h(t, x) \in \mathbb{R}^{+}$, being Lipschitz continuous in the space variable and satisfying a linear growth condition in the space variable uniformly in the time variable

$v(t, x, p):=\inf \left\{y \geqslant 0: \exists(\nu, \alpha) \in \mathcal{U}_{t, x, y} \times \mathcal{A}_{t, p}^{t_{1}}\right.$ s.t. $\left.Y_{t_{1}}^{t, x, y, \nu} \geqslant(h \vee g)\left(t_{1}, X_{t_{1}}^{t, x}, P_{t_{1}}^{t, p, \alpha}\right)\right\}$, with $\mathcal{A}_{t, p}^{t_{1}}$ defined in (3.7) and where $(h \vee g)(t, x, p):=h(t, x) \vee g(t, x, p)$ with $g$ defined in (3.10). Then there exists $\hat{\alpha} \in \mathcal{A}_{t, p}^{t_{1}}$ such that

$$
v(t, x, p)=\mathbb{E}^{\mathbb{Q}_{t, x}}\left[\overline{\operatorname{conv}}(h \vee g)\left(t_{1}, X_{t_{1}}^{t, x}, P_{t_{1}}^{t, p, \hat{\alpha}}\right)\right],
$$

with $\overline{\operatorname{conv}}(h \vee g)$ defined in (3.12) with $f \equiv h$.

Proof. We consider $(t, x) \in\left[t_{0}, t_{1}\right) \times \mathcal{O}_{+}^{d}$ and $p \in[0,1]$, unless otherwise stated.

1. Probabilistic representation of $v$.

1.a Arguing as in [2, proof of Proposition 2.3] one can first prove that

$$
v(t, x, p)=\inf _{\alpha \in \mathcal{A}_{t, p}^{t_{1}}} \mathbb{E}^{\mathbb{Q}_{t, x}}\left[(h \vee g)\left(t_{1}, X_{t_{1}}^{t, x}, P_{t_{1}}^{t, p, \alpha}\right)\right] .
$$

1.b Then following the arguments of [2, proof of Proposition 3.4] and using (3.8)-(3.9) and (7.1) we obtain

$$
v(t, x, p)=\inf _{\alpha \in \mathcal{A}_{t, p}^{t_{1}}} \mathbb{E}^{\mathbb{Q}_{t, x}}\left[\overline{\operatorname{conv}}(h \vee g)\left(t_{1}, X_{t_{1}}^{t, x}, P_{t_{1}}^{t, p, \alpha}\right)\right] .
$$

2. Proof of the existence of $\hat{\alpha} \in \mathcal{A}_{t, p}^{t_{1}}$.

We now introduce the function

$$
w(t, x, q)=\mathbb{E}^{\mathbb{Q}_{t, x}}\left[(h \vee g)^{\sharp}\left(t_{1}, X_{t_{1}}^{t, x}, q Q_{t_{1}}^{t, x, 1}\right)\right]+\infty \mathbf{1}_{\{q<0\}},
$$

where $(h \vee g)^{\sharp}$ stands for the Legendre-Fenchel transform of $(h \vee g)$. We recall that we also denote by $w^{\sharp}$ the dual of $w$.

2.a Appealing to (7.1), the definition of the Legendre-Fenchel transform (recall the Notations), (3.4) and the definition of $\mathcal{A}_{t, p}^{t_{1}}$, we obtain that $v \geqslant w^{\sharp}$ on $\left[t_{0}, t_{1}\right) \times \mathcal{O}_{+}^{d} \times[0,1]$ (see e.g. [2, proof of Proposition 3.1]).

2.b Differentiability of $w(t, x, \cdot)$ on $\mathbb{R}^{+}$.

Consider $q \geqslant 0$. Direct computations lead, with [11, Chapter I, Corollary 4.1] and the definition of $g\left(t_{1}, x, \cdot\right)$ on $\mathbb{R}$, to

$$
(h \vee g)^{\sharp}\left(t_{1}, x, q\right)=(\overline{\operatorname{conv}}(h \vee g))^{\sharp}\left(t_{1}, x, q\right)=\left[q-\left[g\left(t_{1}, x\right)-h\left(t_{1}, x\right)\right]^{+}\right]^{+}-h\left(t_{1}, x\right) .
$$


In particular the function $q \in \mathbb{R} \mapsto w(t, x, q)$ is proper, convex and non-decreasing and $w(t, x, 0)=-\mathbb{E}^{\mathbb{Q} t, x}\left[h\left(t_{1}, X_{t_{1}}^{t, x}\right)\right]$ while $w(t, x, q)=\infty$ for $q<0$. We can now state the subsequent properties, using in particular Lebesgue's Theorem:

(a) $\mathrm{D}_{q}^{+} w(t, x, \cdot) \geqslant 0$ if $q \geqslant 0$ and $\mathrm{D}_{q}^{-} w(t, x, \cdot) \geqslant 0$ if $q>0$,

(b) $\lim _{q \uparrow \infty} \mathrm{D}_{q}^{ \pm} w(t, x, q)=1$,

(c) $\mathrm{D}_{q}^{+} w(t, x, 0)=\mathbb{P}\left[\left[g\left(t_{1}, X_{t_{1}}^{t, x}\right)-h\left(t_{1}, X_{t_{1}}^{t, x}\right)\right]^{+}=0\right]:=p_{\mathrm{h}}(t, x)$,

(d) the sub-differential of $w(t, x, \cdot)$ at $q=0$ belongs to $\left(-\infty, p_{\mathrm{h}}(t, x)\right]$.

Moreover,

$$
\begin{aligned}
\mathrm{D}_{q}^{-} w(t, x, q) & =\mathbb{E}\left[\mathrm{D}_{q}^{-}(h \vee g)^{\sharp}\left(t_{1}, X_{t_{1}}^{t, x}, q Q_{t_{1}}^{t, x, 1}\right)\right] \\
& =\mathbb{P}\left[q Q_{t_{1}}^{t, x, 1}>\left[g\left(t_{1}, X_{t_{1}}^{t, x}\right)-h\left(t_{1}, X_{t_{1}}^{t, x}\right)\right]^{+}\right] \text {for } q>0,
\end{aligned}
$$

and

$$
\begin{aligned}
\mathrm{D}_{q}^{+} w(t, x, q) & =\mathbb{E}\left[\mathrm{D}_{q}^{+}(h \vee g)^{\sharp}\left(t_{1}, X_{t_{1}}^{t, x}, q Q_{t_{1}}^{t, x, 1}\right)\right] \\
& =\mathbb{P}\left[q Q_{t_{1}}^{t, x, 1} \geqslant\left[g\left(t_{1}, X_{t_{1}}^{t, x}\right)-h\left(t_{1}, X_{t_{1}}^{t, x}\right)\right]^{+}\right] \text {for } q \geqslant 0 .
\end{aligned}
$$

2.c Bounds of $w^{\sharp}(t, x, \cdot)$ on its domain.

Appealing to the definition of the Legendre-Fenchel transform, (7.2), the step 2.b and to [20, Theorem 12.2], we deduce that $w^{\sharp}(t, x, \cdot)$ is convex, non-decreasing, non-negative and proper on its domain and continuous on the interior of its domain. Now using the definition of $w$ in (7.2) together with (3.4) and (7.3) and after applying the Monotone Convergence Theorem, we write

$$
\begin{aligned}
w^{\sharp}(t, x, 1)=\sup _{q \geqslant 0}\{q-w(t, x, q)\} \geqslant \lim _{q \rightarrow \infty}\{q-w(t, x, q)\} & =\mathbb{E}^{\mathbb{Q}_{t, x}}\left[(h \vee g)\left(t_{1}, X_{t_{1}}^{t, x}, 1\right)\right] \\
& =v(t, x, 1) .
\end{aligned}
$$

Combining the latter inequality with the step 2.a we finally conclude that $w^{\sharp}(t, x, 1)=$ $v(t, x, 1)$. Finally we note that $w^{\sharp}(t, x, p) \geqslant \lim _{q \rightarrow \infty}\{q(p-1)+q-w(t, x, q)\}=\infty$ for $p>1$, by the non-decreasing property of $q \mapsto q-w(t, x, q)$ (recall the step 2.b). Moreover $w^{\sharp}(t, x, p)=\mathbb{E}^{\mathbb{Q}_{t, x}}\left[h\left(t_{1}, X_{t_{1}}^{t, x}\right)\right]$ for $p \in(-\infty, 0]$ (recall that $w(t, x, 0)=$ $-\mathbb{E}^{\mathbb{Q}_{t, x}}\left[h\left(t_{1}, X_{t_{1}}^{t, x}\right)\right]$ on $\left[t_{0}, t_{1}\right) \times \mathcal{O}_{+}^{d}$ by the step 2.b). Therefore combining the latter results with the non-decreasing property of $w^{\sharp}(t, x, \cdot)$ we conclude that the domain of $w^{\sharp}(t, x, \cdot)$ is $(-\infty, 1]$. Finally appealing again to the non-decreasing property of $w^{\sharp}(t, x, \cdot)$ we get from its closeness that it is continuous on its domain.

2.d Probabilistic representation of $w^{\sharp}$.

Proceeding as in [2, Theorem 3.1] we obtain from the steps 2.b and 2.c the following representation of $w^{\sharp}$. Observe that for the sake of clarity we will not write the 
dependencies of the variables involved in the following discussion. For $p \in\left(p_{\mathrm{h}}(t, x), 1\right)$,

$$
w^{\sharp}(t, x, p)=\mathbb{E}^{\mathbb{Q}_{t, x}}\left[\overline{\operatorname{conv}}(h \vee g)\left(t_{1}, X_{t_{1}}^{t, x}, P_{t_{1}}^{t, p, \hat{\alpha}}\right)\right],
$$

where $\hat{\alpha} \in \mathcal{A}_{t, p}^{t_{1}}$ comes from the Martingale Representation Theorem of

$$
\left(\lambda \mathrm{D}_{q}^{+}(h \vee g)^{\sharp}+(1-\lambda) \mathrm{D}_{q}^{-}(h \vee g)^{\sharp}\right)\left(t_{1}, X_{t_{1}}^{t, x}, \hat{q} Q_{t_{1}}^{t, x, 1}\right)=p+\int_{t}^{t_{1}} \hat{\alpha}_{s}^{\top} \mathrm{d} W_{s}=: P_{t_{1}}^{t, p, \hat{\alpha}},
$$

with $\lambda \in[0,1]$ and $\hat{q}>0$ being such that

$$
p=\mathbb{E}\left[\left(\lambda \mathrm{D}_{q}^{+}(h \vee g)^{\sharp}+(1-\lambda) \mathrm{D}_{q}^{-}(h \vee g)^{\sharp}\left(t_{1}, X_{t_{1}}^{t, x}, \hat{q} Q_{t_{1}}^{t, x, 1}\right)\right] .\right.
$$

Moreover if $p \in\left[0, p_{\mathrm{h}}(t, x)\right]$ then $\hat{q}=0$ and $p=\mathbb{E}\left[\lambda \mathrm{D}_{q}^{+}(h \vee g)^{\sharp}\left(t_{1}, X_{t_{1}}^{t, x}, 0\right)\right], \lambda \in[0,1]$ while, if $p=1$, the result found in the step 2.c gives $w^{\sharp}(t, x, 1)=v(t, x, 1)$ and thus $\hat{\alpha}=0$.

\section{2.e Conclusion.}

First appealing to the steps $1 . \mathrm{b}$ and 2.d we prove that $v \leqslant w^{\sharp}$ on $\left[t_{0}, t_{1}\right) \times \mathcal{O}_{+}^{d} \times[0,1]$ which, combined with the step 2.a, concludes the proof.

\section{References}

[1] Dimitri P Bertsekas and Steven E Shreve. Stochastic Optimal Control: the Discrete Time Case, volume 139. Academic Press New York, 1978.

[2] Bruno Bouchard, Géraldine Bouveret, and Jean-François Chassagneux. A backward dual representation for the quantile hedging of Bermudan options. SIAM Journal on Financial Mathematics, 7(1):215-235, 2016.

[3] Bruno Bouchard, Romuald Elie, and Antony Réveillac. BSDE with weak terminal condition. The Annals of Probability, 43(2):572-604, 2015.

[4] Bruno Bouchard, Romuald Elie, and Nizar Touzi. Stochastic target problems with controlled loss. SIAM Journal on Control and Optimization, 48(5):3123-3150, 2009 .

[5] Bruno Bouchard, Ludovic Moreau, and Marcel Nutz. Stochastic target games with controlled loss. The Annals of Applied Probability, 24(3):899-934, 2014.

[6] Bruno Bouchard and Thanh Nam Vu. The obstacle version of the geometric dynamic programming principle: Application to the pricing of American options under constraints. Applied Mathematics and Optimization, 61(2):235-265, 2010. 
[7] Phelim Boyle and Weidong Tian. Portfolio management with constraints. Mathematical Finance, 17(3):319-343, 2007.

[8] Michael W Brandt and Jules H Van Binsbergen. Optimal asset allocation in assetliability management. Technical report, National Bureau of Economic Research, 2007.

[9] Jérôme Detemple and Marcel Rindisbacher. Dynamic asset-liability management with tolerance for limited shortfalls. Insurance: Mathematics and Economics, 43(3):281-294, 2008.

[10] Roxana Dumitrescu. BSDEs with nonlinear weak terminal condition. arXiv preprint arXiv:1602.00321, 2016.

[11] Ivar Ekeland and Roger Teman. Analyse Convexe et Problèmes Variationnels. Paris Dunod-Gauthier Villars, 1973.

[12] Nicole El Karoui. Les aspects probabilistes du contrôle stochastique. In Ecole d'Eté de Probabilités de Saint-Flour IX-1979, pages 73-238. Springer, 1981.

[13] Nicole El Karoui, Monique Jeanblanc, and Vincent Lacoste. Optimal portfolio management with American capital guarantee. Journal of Economic Dynamics and Control, 29(3):449-468, 2005.

[14] Hans Föllmer and Peter Leukert. Quantile hedging. Finance and Stochastics, 3(3):251-273, 1999.

[15] Hans Föllmer and Peter Leukert. Efficient hedging: cost versus shortfall risk. Finance and Stochastics, 4(2):117-146, 2000.

[16] Ying Jiao, Olivier Klopfenstein, and Peter Tankov. Hedging under multiple risk constraints. Finance and Stochastics, 21(2):361-396, 2017.

[17] Ioannis Karatzas and Steven E Shreve. Brownian Motion and Stochastic Calculus, volume 113. Springer-Verlag New York, 1998.

[18] Lionel Martellini and Vincent Milhau. Dynamic allocation decisions in the presence of funding ratio constraints. Journal of Pension Economics and Finance, 11(04):549-580, 2012.

[19] Ludovic Moreau. Stochastic target problems with controlled loss in jump diffusion models. SIAM Journal on Control and Optimization, 49(6):2577-2607, 2011. 
[20] R Tyrrell Rockafellar. Convex Analysis. Number 28. Princeton university press, 1997.

[21] H Mete Soner and Nizar Touzi. Dynamic programming for stochastic target problems and geometric flows. Journal of the European Mathematical Society, 4(3):201236, 2002.

[22] H Mete Soner and Nizar Touzi. Stochastic target problems, dynamic programming, and viscosity solutions. SIAM Journal on Control and Optimization, 41(2):404$424,2002$. 